\title{
Polynomial Flow-Cut Gaps and Hardness of Directed Cut Problems*
}

\author{
Julia Chuzhoy ${ }^{\dagger} \quad$ Sanjeev Khanna ${ }^{\ddagger}$
}

December 10, 2008

\begin{abstract}
We study the multicut and the sparsest cut problems in directed graphs. In the multicut problem, we are a given an $n$-vertex graph $G$ along with $k$ source-sink pairs, and the goal is to find the minimum cardinality subset of edges whose removal separates all source-sink pairs. The sparsest cut problem has the same input, but the goal is to find a subset of edges to delete so as to minimize the ratio of the number of deleted edges to the number of source-sink pairs that are separated by this deletion. The natural linear programming relaxation for multicut corresponds, by LP-duality, to the well-studied maximum (fractional) multicommodity flow problem, while the standard LP-relaxation for sparsest cut corresponds to maximum concurrent flow. Therefore, the integrality gap of the linear programming relaxation for multicut/sparsest cut is also the flow-cut gap: the largest gap, achievable for any graph, between the maximum flow value and the minimum cost solution for the corresponding cut problem.

Our first result is that the flow-cut gap between maximum multicommodity flow and minimum multicut is $\tilde{\Omega}\left(n^{1 / 7}\right)$ in directed graphs. We show a similar result for the gap between maximum concurrent flow and sparsest cut in directed graphs. These results improve upon a long-standing lower bound of $\Omega(\log n)$ for both types of flow-cut gaps. We notice that these polynomially large flow-cut gaps are in a sharp contrast to the undirected setting where both these flow-cut gaps are known to be $\Theta(\log n)$. Our second result is that both directed multicut and sparsest cut are hard to approximate to within a factor of $2^{\Omega\left(\log ^{1-\epsilon} n\right)}$ for any constant $\epsilon>0$, unless NP $\subseteq$ ZPP. This improves upon the recent $\Omega(\log n / \log \log n)$-hardness result for these problems. We also show that existence of PCP's for NP with perfect completeness, polynomially small soundness, and constant number of queries would imply a polynomial factor hardness of approximation for both these problems. All our results hold for directed acyclic graphs.
\end{abstract}

\footnotetext{
*A preliminary version of this paper appeared in the Proceedings of the 39th Annual ACM Symposium on Theory of Computing, 2007 [14].

${ }^{\dagger}$ Toyota Technological Institute, Chicago, IL 60637. Email: cjulia@tti-c.org. Work done while the author was a member at the School of Mathematics, Institute for Advanced Study. Supported by a grant of the state of New Jersey to the Institute for Advanced Study.

${ }^{\ddagger}$ Dept. of Computer \& Information Science, University of Pennsylvania, Philadelphia, PA 19104. Email: sanjeev@cis. upenn.edu. Supported in part by a Guggenheim Fellowship, an IBM Faculty Award, an NSF Career Award CCR-0093117, and by NSF Award CCF-0635084.
} 


\section{Introduction}

Cuts are fundamental combinatorial objects that play an important role in the study of embeddings, graph theory, Markov chains, parallel computation and optimization. Some representative applications of cuts include the use of bisection width and flux in establishing bounds for parallel algorithms, use of conductance in establishing rapid-mixing property of Markov chains, balanced separators for divide-and-conquer algorithms, and sparsest cuts in network design and routing. Essentially all cut problems that arise in the above-mentioned application scenarios are NP-hard. One of the most successful approaches to designing approximation algorithms for cut problems relies on the rounding of natural linear-programming relaxations for these problems. The dual linear programs of these relaxations formulate flow problems in networks. By strong duality, the largest gap between the maximum flow and the minimum cut achievable for any problem instance (also called the flow-cut gap) is exactly the integrality gap of these cut relaxations. If one views a cut computation as revealing some inherent bottleneck to communication (flow) capacity of a network, then the flow-cut gap may be viewed as revealing how closely this bottleneck can be approached. Starting from the celebrated max-flow min-cut theorem of Ford and Fulkerson, flow-cut gaps have played a central role in combinatorial optimization. For many optimization problems, the best known approximation guarantees correspond to upper bounds on flow-cut gaps. Some examples include approximation guarantees for undirected and directed multicut problems, sparsest cut in planar graphs, well-linked decompositions, and the performance ratio for oblivious routing.

In this paper, we make progress on some long-standing questions concerning flow-cut gaps in directed graphs and approximability of directed cut problems. We start by describing the cut problems that we study with a brief review of prior work for them.

\subsection{Directed Multicut}

An instance of the directed multicut problem consists of a directed $n$-vertex graph $G(V, E)$ and a collection of $k$ source-sink pairs $\left\{\left(s_{1}, t_{1}\right), \ldots,\left(s_{k}, t_{k}\right)\right\}$. The goal is to remove the smallest possible number of edges so as to separate all source-sink pairs; a pair $\left(s_{i}, t_{i}\right)$ is considered separated iff in the resulting graph there is no path connecting $s_{i}$ to $t_{i}$. The parameter $k$ is also referred to as the number of commodities in the instance. Vertices in the set $T=\left\{s_{1}, t_{1}, s_{2}, t_{2}, \ldots, s_{k}, t_{k}\right\}$ are called terminals, and all the other vertices are non-terminals.

For the single-commodity case, the celebrated max-flow min-cut theorem [22] shows that the size of minimum $\left(s_{1}, t_{1}\right)$ cut equals the maximum flow from $s_{1}$ to $t_{1}$. The tight duality between cuts and flows breaks down even in undirected graphs for $k \geq 3$. However, the (worst-case) gap between maximum flow and the minimum multicut is well understood for undirected graphs and is known to be $\Theta(\log k)[31,24]$. In a sharp contrast, Saks et al. [33] have shown that the flow-cut gap in directed graphs can be as large as $k-\epsilon$ for any $\epsilon>0$. Since it is easy to see that the flow-cut gap cannot exceed $k$, it may seem that the flow-cut gaps are well understood in the directed case as well. However, the size of the Saks et al. construction grows super-exponentially in $k$, and the gap realized by these instances is only $O(\log n / \log \log n)$, where $n$ is the number of vertices in $G$. As a function of $n$, the strongest gap known is $\Omega(\log n)$, and it is achieved by an expander-based construction [31]. This lack of understanding of the directed flow-cut gaps is reflected as well in a large separation between the upper and the lower bounds on the approximability threshold of directed multicut. Cheriyan, Karloff and Rabani [12] gave an $O(\sqrt{n \log n})$-approximation algorithm for directed multicut, and Gupta [26] subsequently improved it to an $O(\sqrt{n})$-approximation. Kortsarts,

Kortsarz and Nutov [30] showed an $\tilde{O}\left(n^{2 / 3} / \mathrm{OPT}^{1 / 3}\right)$-approximation, where OPT is the cost of the optimal solution, and more recently, Agarwal, Alon, and Charikar [1] have further improved the 
approximation ratio to $\tilde{O}\left(n^{11 / 23}\right)$. On the hardness front, recently, Chuzhoy and Khanna [13] established an $\Omega(\log n / \log \log n)$-hardness for directed multicut, assuming that NP is not contained in DTIME $\left(n^{\text {polylog(n) }}\right)$. While these algorithmic and hardness results represent important steps in closing the gaps in our understanding of the directed multicut problem, they leave open the possibility that the approximability threshold may range anywhere from logarithmic to polynomial function of the input size. We note that the approximability threshold remains unresolved for undirected multicut problem as well, and it currently lies somewhere between APX-hardness [17] and $\Theta(\log k)$ [31, 24]. However, if one assumes the Unique Games Conjecture of Khot [28], undirected multicut problem can be shown to be hard to approximate to within any constant factor [11, 29].

\subsection{Directed Sparsest Cut}

The input to the directed sparsest cut problem is the same as for multicut, but the objective now is to find a subset $E^{\prime}$ of edges so as to minimize the ratio $\left|E^{\prime}\right| /\left|S_{E^{\prime}}\right|$ where $S_{E^{\prime}}$ is the set of sourcesink pairs, which are disconnected in the graph $G\left(V, E \backslash E^{\prime}\right)$. In general, the notion of a sparsest cut in a graph can be defined in two distinct ways. In one version of the problem, which we refer to as the bipartite sparsest cut, the sparsest cut in a graph is a bipartition of vertices into two sets $S$ and $\bar{S}$ that minimizes the ratio of $|\delta(S, \bar{S})|^{1}$ to $\left|\left\{\left(s_{i}, t_{i}\right) \mid s_{i} \in S, t_{i} \in \bar{S}\right\}\right|$. In the second version, which we refer to as the non-bipartite sparsest cut or simply as the sparsest cut, we seek to minimize the ratio of the number of edges deleted to the resulting number of pairs separated. We note here that the dual of concurrent flow problem corresponds to a relaxation for the non-bipartite sparsest cut problem. In undirected graphs, it is easy to see that the two notions are equivalent. However, in directed graphs, as highlighted in a recent work of Charikar et al. [10], these versions seem to behave quite differently. In particular, using a result of Feige and Kogan [21], it is shown in [10] that bipartite sparsest cut is hard to approximate to within $2^{\Omega\left((\log n)^{\delta}\right)}$ for some $\delta>0$ unless 3SAT has subexponential-time algorithms. Furthermore, this hardness can be strengthened to an $n^{\delta}$-hardness for some $\delta>0$ assuming a hypothesis concerning hardness of random 3SAT, as described by Feige [20]. In contrast, for the directed non-bipartite sparsest cut, so far only an $\Omega(\log n / \log \log n)$-hardness is known, due to Chuzhoy and Khanna [13]. On the positive side, the best currently known approximation ratio for directed non-bipartite sparsest cut is an $\tilde{O}\left(n^{11 / 23}\right)$-approximation due to Agarwal, Alon, and Charikar [1], which improves upon an $O(\sqrt{n})$-approximation, due to Hajiaghayi and Räcke [27]. Thus the present approximability status of both directed multicut and directed non-bipartite sparsest cut is similar.

For undirected graphs, a seminal paper of Leighton and Rao [31] showed an $O(\log n)$-approximation algorithm for sparsest cut. Recently, in a significant progress, this approximation factor has been improved to $O(\sqrt{\log n} \log \log n)[7,5]$. On the negative side, Chawla et al. [11] and Khot and Vishnoi [29] showed that, assuming the Unique Games Conjecture of Khot [28], the non-uniform sparsest cut is hard to approximate within any constant factor. Until recently, not even APXhardness is known for sparsest cut in undirected graphs without relying on the Unique Games Conjecture. In a recent progress, Ambühl et al. showed that unless NP is contained in randomized subexponential time $\left(\cap_{\epsilon>0} \operatorname{BPTIME}\left(2^{n^{\epsilon}}\right)\right)$, there is no polynomial-time approximation scheme for even the uniform version of the undirected sparsest cut problem.

\subsection{Our Results and Techniques}

Our first main result establishes a polynomial lower bound on directed flow-cut gaps.

\footnotetext{
${ }^{1} \delta(S, \bar{S})$ refers to all edges $(x, y)$ in $G$ where $x \in S$ and $y \in \bar{S}$.
} 
Theorem 1.1 The flow-cut gap between maximum multicommodity flow and directed multicut is $\tilde{\Omega}\left(n^{1 / 7}\right)$. The flow-cut gap between maximum concurrent flow and directed (non-bipartite) sparsest cut is also $\tilde{\Omega}\left(n^{1 / 7}\right)$. Both results hold even on directed acyclic graphs.

We give an overview of our techniques for the flow-cut gap results above. We focus on the gap between maximum multicommodity flow and directed multicut. For clarity of exposition, we work with the vertex version of the problem, where the goal is to remove the minimum-cardinality subset of non-terminal vertices that disconnects all the source-sink pairs. A standard transformation allows us to translate a flow-cut gap result for the vertex version to the edge version.

Our starting point is an instance $H$ of the multicut problem that is formed by a union of $k$ graphs $H_{1}, \ldots, H_{k}$ defined over the same set of vertices. Instance $H_{i}$ corresponds to the source-sink pair $s_{i}-t_{i}$ and it is a layered graph with $L$ layers for some parameter $L$. We say that path $P$ connecting a source $s_{i}$ to its sink $t_{i}$ is canonical if it is entirely contained in $H_{i}$. The main property of instance $H$ is that if we are required to eliminate only the canonical paths, then fractional solution that assigns $1 / L$ value to each vertex is a feasible solution of cost $n / L$, while the cost of any integral solution must be at least $\Omega(n)$. The fractional solution above is feasible for the restricted problem because the canonical paths in instance $H$ are long (length at least $L$ ). In order to convert this to a true integrality gap result, we need to rule out "short" non-canonical paths. Towards this end, we define another instance $\mathcal{L}$ of directed multicut, which can also be viewed as a union of $k$ graphs $\mathcal{L}_{1}, \ldots, \mathcal{L}_{k}$. Each $\mathcal{L}_{i}$ is a graph with $O(L)$ layers that contains many source-sink pairs. A path connecting a source-sink pair in instance $\mathcal{L}$ is called canonical if it uses only edges from $\mathcal{L}_{i}$. The main property of graph $\mathcal{L}$ is that while the canonical paths share many vertices, no non-canonical paths exist in the graph. We will refer to graph $\mathcal{L}$ as the labeling scheme. The basic idea of using a labeling scheme to ensure that only canonical paths exist between source-sink pairs was first used by Andrews and Zhang [4] to show hardness of directed congestion minimization. The dependence of the size of the labeling scheme on the parameters $k$ and $L$ is crucial to determining the final gap or hardness result. The scheme in [4] gives a graph $\mathcal{L}$ of size $L^{O(\log k)}$, which is insufficient to obtain polynomial gaps. We present a simple new labeling scheme that results in a graph of size poly $(k, L)$. We note that we use a similar labeling scheme in our parallel result on the hardness of directed routing with congestion [15]. The same labeling scheme has also been used independently by [23] for establishing the hardness of directed routing problems with congestion. A merged version of the results from [15] and [23] appears in [16]. It is worth highlighting an important point of departure from the usage of labeling schemes in context of disjoint path problems. A gap result of $\Omega(f(n))$ for directed multicut necessarily requires that the total number of paths connecting source-sink pairs be exponential in $f(n)$. Otherwise, it is easy to see that the flow-cut gap cannot exceed $o(f(n))$. In particular, given any fractional cut solution, if the number of source-sink paths is $2^{o(f(n))}$, then randomly sampling the edges with probability equal to their fractional weight, and repeating the experiment $o(f(n))$ times will separate all source-sink paths with high probability. Since the cost of an optimal fractional cut solution is same as the optimal fractional flow, this rounding shows that there always exists an integral cut of size at most $o(f(n))$ times the flow value on instances with the above-mentioned property. This is in contrast to disjoint-paths problems in directed graphs where a polynomial-factor hardness can be shown even when each source-sink pair has only one possible path in the input graph [16]. Consequently, we modify our basic labeling scheme to ensure that it does not permit any small integral solution. This transformation preserves the property that no non-canonical source-sink paths exist but introduces short canonical paths. The final step is to appropriately compose together graphs $H$ and $\mathcal{L}$ to create a new instance where all canonical paths are long, no non-canonical paths exist and integral solutions have high cost. The resulting 
instance gives us the desired flow-cut gap.

Our second result shows that the flow-cut gaps above can be extended to almost-polynomial hardness of approximation results for directed multicut and sparsest cut.

Theorem 1.2 The directed multicut problem and the directed (non-bipartite) sparsest cut problem are $2^{\Omega\left(\log ^{1-\epsilon} n\right)}$-hard to approximate for any constant $\epsilon>0$, even on directed acyclic graphs, unless $N P \subseteq Z P P$.

One way to show the hardness result above is by replacing the graph $H$ in the flow-cut gap construction with an appropriate graph encoding the constraints from a Raz verifier. There is a natural way of doing such an encoding by strongly using the projection property of the constraints of the Raz verifier. However, this approach cannot give a polynomial hardness since the size of the constraint satisfaction system associated with Raz verifier grows fast as the soundness decreases, and thus soundness which is polynomially small in the system size cannot be achieved.

In order to go to polynomial hardness, we need to allow encoding of general constraint systems which might not have any analog of the projection property. Using several additional ideas we show that we can create desired encodings even when the underlying constraints lack the projection property. An important consequence of our construction is the following theorem.

Theorem 1.3 If NP has probabilistically checkable proof systems with constant number of queries, proof table entries defined over an alphabet $F$ of polynomial size, logarithmic number of random bits, perfect completeness and polynomially small soundness, then both the directed multicut problem and the directed sparsest cut problem are $n^{\Omega(1)}$-hard to approximate, unless NP $\subseteq$ ZPP.

Existence of PCP's for NP with above properties was first conjectured by Bellare et al. [9]. While the conjecture remains unproven yet, a sequence of papers have made progress towards proving this conjecture. In particular, Dinur et al. [18] have shown that for any $\epsilon>0$, NP has a polynomial-size PCP that queries $O(1)$ variables ranging over an alphabet $F$ with $|F|=2^{\Theta\left(\log ^{1-\epsilon} n\right)}$, has perfect completeness, and achieves soundness $O(1 /|F|)$. Proving the Bellare et. al. conjecture requires pushing this result to $|F|=2^{\Theta(\log n)}$, maintaining perfect completeness, and achieving soundness $O\left(1 /|F|^{\beta}\right)$ for some $\beta>0$.

Our final result is an APX-hardness proof of undirected sparsest cut problem. We note that in [13], we had sketched an APX-hardness proof for undirected sparsest cut that is incorrect. We give here a corrected proof for a strengthened version of the result claimed in [13].

Theorem 1.4 There exists a constant $\epsilon>0$ such that there is no $(1+\epsilon)$-approximation algorithm for the non-uniform sparsest cut problem in planar undirected graphs, unless $\mathrm{P}=\mathrm{NP}$.

We note here that unlike in the case of directed graphs, there is no distinction between the bipartite and non-bipartite version of undirected sparsest cut problem. In particular, given a solution to the non-bipartite sparsest cut problem with sparsity $\alpha$, one can construct in polynomial time a bipartite sparsest cut solution with sparsity at most $\alpha$.

\subsection{Organization}

We start with some preliminaries in Section 2. Sections 3 and 4 are devoted to flow-cut gap results and hardness of approximation results, respectively, for directed multicut and directed sparsest cut. Finally, we conclude with APX-hardness of undirected sparsest cut in Section 5. 


\section{Preliminaries}

\subsection{Linear Programming Formulations}

We start by defining a natural LP relaxation for directed multicut. For each edge $e \in E$, there is an indicator variable $x_{e}$ that represents whether or not $e$ is in the solution. For each source-sink pair $\left(s_{i}, t_{i}\right)$, let $\mathcal{P}_{i}$ be the set of all the paths connecting $s_{i}$ to $t_{i}$. The multicut LP-relaxation and its dual are as follows:

$$
\begin{aligned}
& \text { (LP1-P) } \\
& \min \sum_{e \in E} x_{e} \\
& \text { s.t. } \\
& \begin{array}{ll}
\sum_{e \in p} x_{e} \geq 1 & \forall i: 1 \leq i \leq k, \forall p \in \mathcal{P}_{i} \\
x_{e} \geq 0 & \forall e \in E
\end{array} \\
& \begin{array}{cll} 
& (\mathrm{LP} 1-\mathrm{D}) & \\
\max & \sum_{i=1}^{k} \sum_{p \in \mathcal{P}_{i}} f_{p} & \\
\text { s.t. } & & \\
& \sum_{p: e \in p} f_{p} \leq 1 \quad \forall e \in E \\
& f_{p} \geq 0 & \forall i: 1 \leq i \leq k, \forall p \in \mathcal{P}_{i}
\end{array}
\end{aligned}
$$

Notice that (LP1-D) is equivalent to the maximum multicommodity flow problem, where the goal is to maximize the total flow routed between the source-sink pairs, while the flow routed via any edge cannot exceed 1. From LP-duality, the optimal costs of both linear programs are equal, and thus the integrality gap of (LP1-P) is also the flow-cut gap between maximum multicommodity flow and minimum multicut.

We can similarly define an LP-relaxation for sparsest cut. We use the same notation as for the directed multicut LP-formulation. Consider any solution to the sparsest cut problem. For each edge $e \in E$, let $x_{e}$ denote whether edge $e$ is in the solution, and for each $i: 1 \leq i \leq k$, let $h_{i}$ denote whether the source-sink pair $\left(s_{i}, t_{i}\right)$ is disconnected. Let $D=\sum_{i=1}^{k} h_{i}$ be the total number of source-sink pairs disconnected by the solution. We now define, for each edge $e, x_{e}^{\prime}=x_{e} / D$, and for each source-sink pair $\left(s_{i}, t_{i}\right), h_{i}^{\prime}=h_{i} / D$. It is then easy to see that we have defined a feasible solution to the linear program (LP2-P) that appears below, along with its dual (LP2-D). Moreover, the sparsity of the cut equals to the value of the objective function of (LP2-P) on the above solution. Thus (LP2-P) is a relaxation of the directed sparsest cut problem.

$$
\begin{aligned}
& \text { (LP2-P) } \\
& \min \sum_{e \in E} x_{e}^{\prime} \quad \max \lambda \\
& \text { s.t. } \quad \text { s.t. }
\end{aligned}
$$

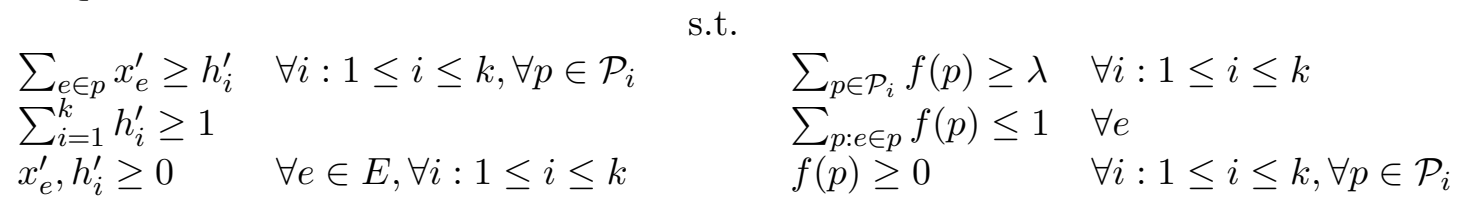

$$
\begin{aligned}
& (\mathrm{LP} 2-\mathrm{D})
\end{aligned}
$$

Notice that (LP2-D) is equivalent to the maximum concurrent flow problem, in which we need to maximize a value $\lambda$, such that $\lambda$ units of flow can be routed simultaneously for each source-sink pair, while the flow on any edge does not exceed 1. From LP-duality, the maximum concurrent flow in any graph is equal to the minimum fractional sparsest cut. Therefore, the integrality gap of (LP2-P) is also the flow-cut gap between maximum concurrent flow and minimum sparsest cut.

\section{$2.2 \quad$ Vertex Version of Multicut}

In establishing our integrality gap and hardness result, we will find it more convenient to work with the vertex version of the directed multicut problem. The input for this problem is denoted by $G=(V, \mathcal{M}, E)$, where $V$ is the set of non-terminal vertices, $\mathcal{M}$ is the set of the source-sink pairs, and $E$ is the set of edges. Let $T(\mathcal{M})=\{s, t \mid(s, t) \in \mathcal{M}\}$ denote the set of all the terminals. The 
goal is to remove the minimum cardinality subset of non-terminal vertices so as to disconnect all the source-sink pairs. Any lower bound on the flow-cut gap or any hardness of approximation result for vertex version of directed multicut can be extended to the (edge) directed multicut problem by a standard transformation. Given an instance $G$ of the vertex version of directed multicut, we replace each non-terminal vertex $v \in V$ by a special directed edge $\left(v^{+} \rightarrow v^{-}\right)$. Each edge $e=(u \rightarrow v)$ in the original graph is now replaced by an edge $\left(u^{-} \rightarrow v^{+}\right)$. Let $G^{\prime}$ denote the new instance. We can assume w.l.o.g., that any integral solution of $G^{\prime}$ only contains special edges, and thus the integral solution costs for both instances are the same. Moreover, if there is a subset of non-terminal vertices in $G$ whose removal disconnects an $\alpha$-fraction of source-sink pairs, then there is also a subset of edges in $G^{\prime}$ of the same size whose removal disconnects the same fraction of source-sink pairs, and vice versa. The linear programming formulation for the vertex version is as follows:

$$
\begin{array}{lll} 
& \text { (LP3) } & \\
\min & \sum_{v \in V} x_{v} & \\
\text { s.t. } & & \\
& \sum_{v \in p \cap V} x_{v} \geq 1 & \forall i: 1 \leq i \leq k, \forall p \in \mathcal{P}_{i} \\
& x_{v} \geq 0 & \forall v \in V
\end{array}
$$

Given a fractional solution to (LP3) on graph $G$, there is a fractional solution of the same value to (LP1-P) on $G^{\prime}$ : each special edge $e=\left(v^{+} \rightarrow v^{-}\right)$is assigned $x_{e}=x_{v}$. It follows that the integrality gap and hardness results for the vertex version of multicut carry over to our original problems. This is also true for the bi-criteria setting, where only a constant fraction of source-sink pairs needs to be disconnected.

\section{Integrality (Flow-Cut) Gap Results for Directed Cut Problems}

In this section, we will establish Theorem 1.1. We will use the phrases integrality gap and flow-cut gap interchangeably throughout this section. We will start by establishing the flow-cut gap result for the directed multicut problem. As indicated in Section 2.2, we will work with the vertex version of directed multicut without any loss of generality. We will show that the flow-cut gap holds even when the fractional solution is required to disconnect all source-sink pairs while the integral solution is required to disconnect only a $(1-\epsilon)$-fraction of pairs, for some specific $\epsilon>0$. A simple argument is then used to translate this "bi-criteria" flow-cut gap result to a flow-cut gap result for directed sparsest cut.

\subsection{Flow-Cut Gap Construction for Directed Multicut}

\subsubsection{Overview}

We start with a brief overview of construction. Let $n$ be some parameter. We construct a (vertex) multicut instance $G$ where all the parameters are defined in terms of $n$, and the instance size (the number of vertices) is $N=O\left(n^{7} /(\log n)^{3}\right)$. We show that the integrality gap of $G$ is $\Omega\left(\frac{n}{\log n}\right)=$ $\Omega\left(\frac{N^{1 / 7}}{\log ^{4 / 7} N}\right)$.

Let $L=n /(4 \log n)$. Our goal is to construct a multicut instance $G$ that has the following properties: $\left(\mathbf{C}_{1}\right)$ : Any path connecting any source-sink pair contains at least $L$ non-terminal vertices.

$\left(\mathbf{C}_{2}\right)$ : There exists a constant $0<\epsilon<1$ such that any integral solution that disconnects at least $(1-\epsilon)$-fraction of the pairs contains at least $\Omega(N)$ vertices. 
It is easy to see that if $G$ has the above properties, then the integrality gap is at least $\Omega(L)=$

$\Omega\left(\frac{N^{1 / 7}}{\log ^{4 / 7} N}\right)$, since a feasible fractional solution to (LP3) of cost $O(N / L)$ can be obtained by assigning $1 / L$-fraction to each non-terminal vertex.

The graph $G$ is constructed in three steps. In the first step, we construct an initial multicut instance $H$, and we define, for each source-sink pair, a collection of canonical paths. Graph $H$ has property $\left(C_{2}\right)$, while property $\left(C_{1}\right)$ holds for canonical paths, i.e., each canonical path contains at least $L$ non-terminal vertices. However, there are also non-canonical paths connecting source-sink pairs in $H$, which might contain few non-terminal vertices. Thus, property $\left(C_{1}\right)$ does not hold in general. The goal of the next two steps is to eliminate the non-canonical paths, while preserving the other properties of $H$. To achieve this, in the second step we construct a graph $\mathcal{L}$, called the labeling scheme. Graph $\mathcal{L}$ also has a collection of source-sink pairs, and for each source-sink pair a collection of canonical paths connecting the source to the sink is defined. The main property of graph $\mathcal{L}$ is that while the canonical paths share many vertices, no non-canonical source-sink paths exist in $\mathcal{L}$. In the third step, we compose the graphs $H$ and $\mathcal{L}$ together to obtain the final graph $G$, for which both properties $\left(C_{1}\right)$ and $\left(C_{2}\right)$ are true.

\subsubsection{Step 1: Constructing Graph $H$}

In this section we construct our initial multicut instance $H=(V, \mathcal{M}, E)$. Let $V=\{1, \ldots, n\}$ be the set of non-terminal vertices of $H$, and let $k=n$. For each $i: 1 \leq i \leq k$, we have a source-sink pair $s_{i}$ - $t_{i}$. Thus, $\mathcal{M}=\left\{\left(s_{i}, t_{i}\right) \mid 1 \leq i \leq k\right\}$. In order to define set $E$ of edges, we construct, for each source-sink pair $s_{i}$ - $t_{i}$, an auxiliary graph $H_{i}$, which is defined over the same set of vertices as $H$. Graph $H$ can then be viewed as the union of graphs $H_{i}, 1 \leq i \leq k$. Thus, if we denote by $E_{i}$ the set of edges of graph $H_{i}$, then $E=\cup_{i=1}^{k} E_{i}$. We call the edges belonging to set $E_{i}$ edges of type $i$.

The Graph $H_{i}$ : Fix some $i: 1 \leq i \leq k$. Graph $H_{i}$ contains a single source-sink pair $\left(s_{i}, t_{i}\right)$. The non-terminal vertices of $H_{i}$ are a subset of $V$ and they are arranged into $L=n /(4 \log n)$ layers containing $\lambda=\log n$ vertices each. The layers are denoted $S_{1}(i), S_{2}(i), \ldots, S_{L}(i) \subseteq V$ and they are constructed one after another, starting from the first layer. In order to construct the $j$ th layer, for $1 \leq j \leq L$, we select uniformly at random $\lambda$ distinct vertices from set $V \backslash\left(S_{1}(i) \cup \cdots \cup S_{j-1}(i)\right)$. Notice that since $\lambda=\log n, L=n /(4 \log n)$ and $|V|=n$, it is possible to construct these layers, and in total $\left|\bigcup_{j=1}^{L} S_{j}(i)\right|=n / 4$. Edges $E_{i}$ of graph $H_{i}$ are defined as follows. There is an edge from $s_{i}$ to every vertex in the first layer, $S_{1}(i)$. For every pair of consecutive layers $S_{j}(i), S_{j+1}(i)$, where $j: 1 \leq j<L$, there is an edge from every vertex in $S_{j}(i)$ to every vertex in $S_{j+1}(i)$. Finally, for every vertex in the last layer $S_{L}(i)$, there is an edge connecting this vertex to $t_{i}$. This concludes the definition of graph $H_{i}$. Recall that the final set of edges of graph $H$ is $E=\cup_{i=1}^{k} E_{i}$, where the edges in set $E_{i}$ are called edges of type $i$.

Properties of graph $H$ : A path connecting a source $s_{i}$ to sink $t_{i}$ (for $1 \leq i \leq k$ ) is called a canonical path if it only contains edges of type $i$.

Observation 1 The number of non-terminal vertices on any canonical source-sink path in graph $H$ is at least $L$.

The observation follows from the fact that any canonical path connecting $s_{i}$ to $t_{i}$ in $H$ also exists in graph $H_{i}$, and after leaving source $s_{i}$ it has to traverse all the layers of graph $H_{i}$ before reaching sink $t_{i}$. Therefore, property $\left(C_{1}\right)$ holds for canonical paths. However, there might also be non-canonical paths connecting $s_{i}$ to $t_{i}$ whose length can be short and for which $\left(C_{1}\right)$ is not true. 
Next we establish property $\left(C_{2}\right)$ for graph $H$. We actually establish a stronger version of this property that we need for future analysis. Let $\mathcal{S} \subseteq V$ be any subset of non-terminal vertices, $|\mathcal{S}| \leq n / 16$. For each $i: 1 \leq i \leq k$, we say that $i$ is covered by $\mathcal{S}$, iff the removal of vertices of $\mathcal{S}$ from graph $H_{i}$ disconnects $s_{i}$ from $t_{i}$ in this graph. Equivalently, $\mathcal{S}$ covers $i$ iff there exists a layer $j: 1 \leq j \leq L$ with $S_{j}(i) \subseteq \mathcal{S}$. Let $\mathcal{B}$ be the following bad event: there is a set $\mathcal{S} \subseteq V$ of non-terminal vertices, $|\mathcal{S}| \leq n / 16$, such that $\mathcal{S}$ covers more than half the indices $i: 1 \leq i \leq k$. The next lemma bounds the probability of event $\mathcal{B}$.

Lemma 3.1 The probability of event $\mathcal{B}$ is at most $2^{-n}$.

Proof: Let $\mathcal{S} \subseteq V$ be any subset of non-terminal vertices, $|\mathcal{S}| \leq n / 16$. Fix some $i: 1 \leq i \leq k$ and consider the random choices made when layers $S_{1}(i), \ldots, S_{L}(i)$ of graph $H_{i}$ are constructed. Even though the choices are not independent, when vertices of subset $S_{j}(i)$ are chosen, the size of the set $V \backslash \bigcup_{\ell=1}^{j-1} S_{\ell}(i)$ is at least $\frac{3 n}{4}+\lambda$. Therefore, no matter what vertices have been chosen by $S_{1}(i), \ldots, S_{j-1}(i)$, the probability that $S_{j}(i) \subseteq \mathcal{S}$ is at $\operatorname{most}\left(\frac{n / 16}{3 n / 4}\right)^{\lambda} \leq\left(\frac{1}{8}\right)^{\log n} \leq \frac{1}{n^{3}}$ (we use conditional probabilities). Therefore, using the union bound, the probability that $i$ is covered by $\mathcal{S}$ is at most $\frac{L}{n^{3}} \leq \frac{1}{4 n^{2} \log n}$. Since the random choices made for different graphs $H_{i}, 1 \leq i \leq k$ are completely independent, the probability that half of these indices are covered is at most:

$$
\left(\begin{array}{c}
n \\
n / 2
\end{array}\right)\left(\frac{1}{4 n^{2} \log n}\right)^{n / 2} \leq 2^{-n \log n / 4}
$$

The number of possible choices of subset $\mathcal{S}$ is at most $2^{n}$, and applying the union bound for all such subsets finishes the proof.

Let $\mathcal{S} \subseteq V$ be any subset of non-terminal vertices. Observe that if $\mathcal{S}$ disconnects a source-sink pair $s_{i}$ - $_{i}$ in graph $H$, then $\mathcal{S}$ also covers $i$. Therefore, from Lemma 3.1, any solution of size up to $n / 16$ disconnects at most half the source-sink pairs in $H$, with high probability. From now on we assume that $\mathcal{B}$ does not happen.

As we have shown, property $\left(C_{2}\right)$ holds in graph $H$ with high probability. As for property $\left(C_{1}\right)$, we are only guaranteed that it holds for the canonical paths. It is therefore possible that for many source-sink pairs short non-canonical paths exist. The goal of the next steps is to resolve this problem while preserving the other properties of graph $H$.

\subsubsection{Step 2: Handling Non-Canonical Paths}

In this section we build a graph $\mathcal{L}$ that represents the labeling scheme. In the final step we combine graphs $H$ and $\mathcal{L}$ together to obtain the final graph $G$. We notice that we use the labeling scheme and its associated graph in the hardness of approximation construction as well. We start with the definition of a labeling scheme.

A labeling scheme with parameters $\tau$ and $Z$, is denoted by $\mathcal{L}=\mathcal{L}_{\tau, Z}=\left(U, \mathcal{M}^{\prime}, E^{\prime}\right)$, and it is defined as follows. There is a set $Y=\{1, \ldots,|Y|\}$ of labels associated with the labeling scheme. The nonterminal vertices are partitioned into $Z$ layers, where each layer contains one vertex representing each label $y \in Y$. Thus, the set of the non-terminal vertices is: $U=\{u(y, h) \mid y \in Y, 1 \leq h \leq Z\}$. There are $\tau$ different types of source-sink pairs. For each $i: 1 \leq i \leq \tau$, there are $|Y|$ sources of type $i:\left\{s_{i}(y)\right\}_{y \in Y}$, and $|Y|$ sinks of type $i:\left\{t_{i}(y)\right\}_{y \in Y}$. We describe below how these sources and sinks are paired with each other. We now proceed to describe the edges of $\mathcal{L}$. Each edge either connects a source to a vertex in the first layer, or connects a vertex in the last layer to a sink, or connects a non-terminal vertex in layer $j$ to a non-terminal vertex in layer $j+1$, for $1 \leq j<Z$. 
For each $i: 1 \leq i \leq \tau$, there is a set $E_{i}^{\prime}$ of edges of type $i$, which are defined as follows. We define a permutation $\pi_{i}: Y \rightarrow Y$. For each type- $i$ source $s_{i}(y)$, we add an edge from $s_{i}(y)$ to the first-layer vertex $u\left(\pi_{i}(y), 1\right)$. For each non-terminal vertex $u(y, h)$ where $1 \leq h<Z$, we add an edge from $u(y, h)$ to $u\left(\pi_{i}(y), h+1\right)$. Finally, for each layer- $Z$ non-terminal vertex $u(y, Z)$, we add an edge from $u(y, Z)$ to sink $t_{i}\left(\pi_{i}(y)\right)$. Therefore, edges of type $i$ form a perfect matching between each pair of consecutive layers, between the type- $i$ sources and the vertices of the first layer, and between the vertices of the last layer and the type- $i$ sinks. Thus, if we start at some type- $i$ source $s_{i}(y)$ and follow type- $i$ edges, then we will construct a path, denoted by $P_{i}(y)$, that ends at some type- $i$ sink $t_{i}\left(y^{\prime}\right)$. Moreover, the paths $\left\{P_{i}(y)\right\}_{y \in Y}$ are vertex disjoint. The pairs of endpoints of these paths define the source-sink pairs for the labeling scheme. Thus, we have $|Y|$ source-sink pairs of type $i$, and each type- $i$ source and sink is involved in exactly one such pair. The paths $P_{i}(y)$ are called the canonical paths for the corresponding source-sink pairs. Notice that for each source-sink pair there is a unique canonical path connecting it.

A labeling scheme $\mathcal{L}$ is called valid iff for every source-sink pair, the canonical path is the only path connecting the source to the sink. In other words, no non-canonical paths connecting sourcesink pairs exist. We note that the construction of [4] can be viewed as a labeling scheme, where $|Y|=Z^{O(\log \tau)}$. This construction is insufficient to get a polynomial integrality gap. Below we define a more efficient labeling scheme, where $|Y|=\operatorname{poly}(\tau, Z)$.

In order to define the labeling scheme, we now only need to specify the value of parameter $|Y|$ and to define the permutations $\pi_{i}$, for $1 \leq i \leq \tau$. For each $i: 1 \leq i \leq \tau$, we define an increment vector $\mu_{i} \in \mathbb{Z}^{2}, \mu_{i}=\left(i, i^{2}\right)$. We view the set of labels $Y$ in the following fashion: each label $y \in Y$ is viewed as a 2 -dimensional vector, whose first entry ranges in $[2 \tau Z]$ and the second entry ranges over $\left[2 \tau^{2} Z\right]$. Thus, $Y=[2 \tau Z] \times\left[2 \tau^{2} Z\right]$, and $|Y|=O\left(\tau^{3} Z^{2}\right)$. For each label $y \in Y$, we denote by $y_{1}$ and $y_{2}$ its first and second coordinate respectively. We define an addition operation between pairs of labels (since all increment vectors $\mu_{i} \in Y$, this also defines addition of increment vectors and labels). For $y, y^{\prime} \in Y$, we say that $y \oplus y^{\prime}=y^{\prime \prime}$, iff $y_{1}^{\prime \prime}=y_{1}+y_{1}^{\prime} \bmod (2 \tau Z)$ and $y_{2}^{\prime \prime}=y_{2}+y_{2}^{\prime} \bmod \left(2 \tau^{2} Z\right)$. Finally, we define the permutation $\pi_{i}: Y \rightarrow Y$ for each $i: 1 \leq i \leq k$, as follows: for each $y \in Y$, $\pi_{i}(y)=y \oplus \mu_{i}$. This completes the definition of the labeling scheme. It now only remains to show that the above labeling scheme is valid, i.e., no non-canonical source-sink paths exist. Notice that for each type $i: 1 \leq i \leq \tau, s_{i}(y)-t_{i}\left(y^{\prime}\right)$ are a source-sink pair iff $y^{\prime}=y \oplus\left((Z+1) \mu_{i}\right)$. Thus, the set of source-sink pairs is: $\mathcal{M}^{\prime}=\left\{\left(s_{i}(y), t_{i}\left(y^{\prime}\right)\right) \mid 1 \leq i \leq \tau, y \in Y, y^{\prime}=y \oplus\left((Z+1) \mu_{i}\right)\right\}$.

Lemma 3.2 Let $\left(s_{i}(y), t_{i}\left(y^{\prime}\right)\right) \in \mathcal{M}^{\prime}$ be any source-sink pair of $\mathcal{L}$. Then the only path connecting $s_{i}(y)$ to $t_{i}(y)$ in the graph is the canonical path $P_{i}(y)$.

Proof: Assume otherwise, and let $P$ be a non-canonical path connecting $s_{i}(y)$ to $t_{i}\left(y^{\prime}\right)$. Recall that $y^{\prime}=y \oplus\left((Z+1) \cdot \mu_{i}\right)$. Let $j_{1}, \ldots, j_{Z+1}$ be the types of edges used along path $P$. Since path $P$ is non-canonical, at least one of the types $j_{q} \neq i$, where $1 \leq q \leq Z+1$. As path $P$ must reach the sink $t_{i}\left(y^{\prime}\right)$, it must be the case that $y^{\prime}=y \oplus \mu_{j_{1}} \oplus \cdots \oplus \mu_{j_{Z+1}}$. Recall that the first coordinate in each increment vector lies between 1 and $\tau$, while the second coordinate lies between 1 and $\tau^{2}$. Since the addition of the first coordinate is performed modulo $2 \tau Z$ and the addition of the second coordinate is performed modulo $2 \tau^{2} Z$, we have that $(Z+1) \mu_{i}=\sum_{q=1}^{Z+1} \mu_{j_{q}}$ (here we use standard addition). Therefore, $\mu_{i}$ is convex combination of $\mu_{j_{1}}, \ldots, \mu_{j_{Z+1}}$, while for some $q: 1 \leq q \leq Z+1$, $\mu_{j_{q}} \neq \mu_{i}$. However, the curve $\left(x, x^{2}\right)$ is strictly convex. Therefore, it is impossible that one point on this curve is a convex combination of other points.

We have completed the construction of the labeling scheme $\mathcal{L}$. The main feature of the labeling scheme is that while the canonical paths connecting the source-sink pairs are long and they share 
many vertices, no non-canonical source-sink paths exist in $\mathcal{L}$. There remains however one major obstacle in combining the labeling scheme with graph $H$ to obtain the final graph $G$. When viewed as a directed multicut instance, $\mathcal{L}$ has a cheap solution: the removal of all the vertices in one of the layers disconnects all the source-sink pairs. We perform one final transformation to obtain the final labeling scheme $\mathcal{L}^{\prime}=\left(U, \mathcal{M}^{\prime}, E^{\prime \prime}\right)$. The set of non-terminal vertices and the source-sink pairs in $\mathcal{L}^{\prime}$ are the same as in $\mathcal{L}$. As for the set $E^{\prime \prime}$ of edges, it is defined as follows. For every $i: 1 \leq i \leq k$, we define a set $E_{i}^{\prime \prime}$ of edges of type $i$. Consider any pair of vertices $x, x^{\prime} \in U \cup T\left(\mathcal{M}^{\prime}\right)$ (they can be either terminal or non-terminal vertices). If there is a path consisting of type- $i$ edges from $x$ to $x^{\prime}$ in $\mathcal{L}$, and if $\left(x, x^{\prime}\right) \notin \mathcal{M}^{\prime}$ (i.e., they are not a source-sink pair), then we add a type- $i$ edge $\left(x \rightarrow x^{\prime}\right)$ to $E_{i}^{\prime \prime}$. The final set of edges $E^{\prime \prime}=\cup_{i=1}^{k} E_{i}^{\prime \prime}$. We refer to $\mathcal{L}^{\prime}$ as the modified labeling scheme. Next we describe its properties.

Let $\left(s_{i}(y), t_{i}\left(y^{\prime}\right)\right) \in \mathcal{M}^{\prime}$ be any source-sink pair of type $i$, and let $P$ be any path connecting $s_{i}(y)$ to $t_{i}\left(y^{\prime}\right)$ in $\mathcal{L}^{\prime}$. Path $P$ is called canonical if it contains type- $i$ edges only. Notice that now for each source-sink pair there are many canonical paths.

Claim 3.1 There are no non-canonical source-sink paths in $\mathcal{L}^{\prime}$.

Proof: Let $P$ be any non-canonical path connecting some source-sink pair $s_{i}(y)-t_{i}\left(y^{\prime}\right)$. Let $e=$ $\left(x, x^{\prime}\right)$ be any edge on path $P$, and suppose its type is $i^{\prime}$. Then there is a path connecting $x$ to $x^{\prime}$ in graph $\mathcal{L}$ containing type- $i^{\prime}$ edges only. Therefore, path $P$ corresponds to some non-canonical path connecting $s_{i}(y)$ to $t_{i}\left(y^{\prime}\right)$ in graph $\mathcal{L}$, which is impossible from Lemma 3.2.

\subsubsection{Step 3: the Final Graph}

We are now ready to construct the final graph $G=\left(V^{*}, \mathcal{M}^{*}, E^{*}\right)$, by combining graph $H=$ $(V, \mathcal{M}, E)$ with the modified labeling scheme $\mathcal{L}^{\prime}=\left(U, \mathcal{M}^{\prime}, E^{\prime \prime}\right)$. We use the modified labeling scheme with parameters $\tau=k$ and $Z=32 L$. Thus, $|Y|=O\left(k^{3} Z^{2}\right)=O\left(n^{3} Z^{2}\right)$. The final graph is defined as follows. The set of non-terminal vertices $V^{*}=U \times V$, i.e.,

$$
V^{*}=\{v(y, h, p) \mid y \in Y, 1 \leq h \leq Z, 1 \leq p \leq n\}
$$

For each non-terminal vertex $v(y, h, p)$, we define its pre-images in graphs $H$ and $\mathcal{L}^{\prime}$ to be $g_{H}(v(y, h, p))=$ $p$ and $g_{\mathcal{L}^{\prime}}(v(y, h, p))=u(y, h)$, respectively. The set of source-sink pairs is $\mathcal{M}^{*}=\mathcal{M}^{\prime}$, i.e., $\mathcal{M}^{*}=\left\{\left(s_{i}(y), t_{i}\left(y^{\prime}\right)\right) \mid 1 \leq i \leq k, y \in Y, y^{\prime}=y \oplus\left((Z+1) \mu_{i}\right)\right\}$. The pre-images of the sources in graphs $H$ and $\mathcal{L}^{\prime}$ are defined to be $g_{H}\left(s_{i}(y)\right)=s_{i}$ and $g_{\mathcal{L}^{\prime}}\left(s_{i}(y)\right)=s_{i}(y)$, respectively. The preimages of the sinks are defined in a similar way. Finally, the edges are defined as follows. For each $i: 1 \leq i \leq k$, we define a subset $E_{i}^{*}$ of edges of type $i$, and we set $E^{*}=\bigcup_{i=1}^{n} E_{i}^{*}$. We add a type- $i$ edge $\left(x \rightarrow x^{\prime}\right)$ to set $E_{i}^{*}$ iff a type- $i$ edge exists both in graph $H$ and in graph $\mathcal{L}^{\prime}$ between the corresponding pre-images, i.e.,:

$$
E_{i}^{*}=\left\{\left(x \rightarrow x^{\prime}\right) \mid x, x^{\prime} \in V^{*} \cup T\left(\mathcal{M}^{*}\right),\left(g_{H}(x) \rightarrow g_{H}\left(x^{\prime}\right)\right) \in E_{i} \text { and }\left(g_{\mathcal{L}^{\prime}}(x) \rightarrow g_{\mathcal{L}^{\prime}}\left(x^{\prime}\right)\right) \in E_{i}^{\prime \prime}\right\}
$$

This completes the definition of the graph $G$. The number of non-terminal vertices in the graph $G$ is bounded by $N \leq|Y| Z n \leq O\left(n^{3} L^{3} n\right)=O\left(n^{7} /(\log n)^{3}\right)$. Since $L=n /(4 \log n)$, we have that $L=\Omega\left(\frac{N^{1 / 7}}{(\log N)^{4 / 7}}\right)$.

\subsubsection{The Flow-Cut Gap Analysis}

We next analyze the gap between the costs of the fractional and integral solutions. 
Fractional solution: For any source-sink pair $\left(s_{i}(y), t_{i}\left(y^{\prime}\right)\right) \in \mathcal{M}^{*}$, we say that path $P$ connecting the source to the sink is canonical iff it uses edges of type $i$ only. The next lemma establishes the property $\left(C_{1}\right)$ for the graph $G$.

Lemma 3.3 For any source-sink pair $s_{i}(y)-t_{i}\left(y^{\prime}\right)$ in $G$, any path connecting $s_{i}(y)$ to $t_{i}\left(y^{\prime}\right)$ contains at least $L$ non-terminal vertices.

Proof: The proof relies on the following claim.

Claim 3.2 No non-canonical source-sink paths exist in the graph $G$.

Proof: Assume otherwise. Let $P$ be a non-canonical path connecting some source $s_{i}(y)$ to its sink $t_{i}\left(y^{\prime}\right)$. Let $P^{\prime}$ be the sequence of pre-images of vertices of $P$ in graph $\mathcal{L}^{\prime}$, appearing in the same order as in $P$. Then $P^{\prime}$ forms a non-canonical path that connects the source-sink pair $s_{i}(y)-t_{i}\left(y^{\prime}\right)$ in graph $\mathcal{L}^{\prime}$, which is impossible due to Claim 3.1

We can now complete the proof. Consider any source-sink pair $\left(s_{i}(y), t_{i}\left(y^{\prime}\right)\right) \in \mathcal{M}^{*}$, and let $P$ be any path connecting $s_{i}(y)$ to $t_{i}\left(y^{\prime}\right)$ in $G$. By Claim Lemma 3.2, $P$ must be a canonical path. Let $P^{\prime}$ be a sequence of vertices containing the pre-images of vertices on path $P$ in $H$, in the same order as they appear in $P$. Clearly, $P^{\prime}$ is a canonical path connecting $s_{i}$ to $t_{i}$ in graph $H$ and thus, from Observation 1 , it contains at least $L$ non-terminal vertices. It follows that $P$ also contains at least $L$ non-terminal vertices.

Lemma 3.3 implies that there is a feasible fractional solution of cost $N / L$, achieved by assigning $1 / L$-fraction to each non-terminal vertex of $G$.

Integral solution: A key property of our final construction is summarized by the lemma below.

Lemma 3.4 Assume that event $\mathcal{B}$ does not happen for $H$. Then for any subset $\mathcal{S} \subseteq V^{*}$ of nonterminal vertices, $|\mathcal{S}| \leq N / 32$, the fraction of source-sink pairs which are disconnected when $\mathcal{S}$ is removed from $G$ is at most $99 / 100$.

Proof: Let $\mathcal{S} \subseteq V^{*}$ be any subset of non-terminal vertices, $|\mathcal{S}| \leq N / 32$. For each label $y \in Y$ and for each layer $h: 1 \leq h \leq Z$, let $\mathcal{S}_{y, h}=\{p: v(y, h, p) \in \mathcal{S}\}$. We say that label-layer pair $(y, h)$ is good iff $\left|\mathcal{S}_{y, h}\right| \leq n / 16$. Clearly, at least half the label-layer pairs are good: otherwise, we have $Z|Y| / 2=32 L|Y| / 2$ non-good label-layer pairs, each of them contributing more than $n / 16$ vertices to $\mathcal{S}$, contradicting the fact that $|\mathcal{S}| \leq N / 32=32 L|Y| n / 32$.

Fix a good label-layer pair $(y, h)$. We say that index $i: 1 \leq i \leq n$ is covered at $(y, h)$ iff there is some $j: 1 \leq j \leq L$, such that $S_{j}(i) \subseteq \mathcal{S}_{y, h}$ (recall that $S_{j}(i)$ is the $j$ th layer in graph $\left.H_{i}\right)$. Since we assume that $\mathcal{B}$ does not happen, at least half the indices $i: 1 \leq i \leq n$ are not covered at $(y, h)$.

Let $J \subseteq[1, \ldots, k]$ be the set of all indices $i: 1 \leq i \leq k$, such that the number of label-layer pairs $(y, h)$ for which $i$ is not covered at $(y, h)$ is at least $4|Y| L$.

Claim $3.3|J| \geq k / 7$.

Proof: For each $i \in J$, there are at most $Z|Y|=32|Y| L$ pairs $(y, h)$ that do not cover $i$. For each $i \notin J$, there are at most $4|Y| L$ pairs $(y, h)$ that do not cover $i$. Therefore, in total, the number of triples $(y, h, i)$, where $y \in Y, 1 \leq h \leq Z$ and $1 \leq i \leq k$ and $(y, h)$ does not cover $i$ is at most:

$$
|J| \cdot 32|Y| L+(k-|J|) \cdot 4|Y| L
$$


On the other hand, there are at least $16|Y| L$ good label-layer pairs $(y, h)$, and for each of them at least $k / 2$ indices $i: 1 \leq i \leq k$ are not covered at $(y, h)$. Therefore, we have that:

$$
|J| \cdot 32|Y| L+(k-|J|) \cdot 4|Y| L \geq 8|Y| L k
$$

It follows that $|J| \geq k / 7$.

For each $i: 1 \leq i \leq k$, we call the source-sink pairs in set $\left\{\left(s_{i}(y), t_{i}\left(y^{\prime}\right)\right) \mid y \in Y, y^{\prime}=y \oplus\left((32 L+1) \mu_{i}\right)\right\}$ source-sink pairs of type $i$. The next claim will finish the proof.

Claim 3.4 For each $i \in J$, the fraction of source-sink pairs of type $i$ that are not disconnected by $\mathcal{S}$ is at least $3 / 31$. Therefore, in total, the fraction of source-sink pairs which are not disconnected by $\mathcal{S}$ is at least $\frac{3}{31} \cdot \frac{1}{7} \geq \frac{1}{100}$

Proof: Fix some $i \in J$. We partition the set $Y \times\{1, \ldots, 32 L\}$ of label-layer pairs into $|Y|$ subsets. For each $y \in Y$, we define a subset $T_{y}$, which contains, for each layer $h: 1 \leq h \leq 32 L$, the pair $\left(y_{h}, h\right)$, where $y_{h}=y \oplus\left(h \mu_{i}\right)$. Observe that $\left\{T_{y}\right\}_{y \in Y}$ is indeed a partition of all the label-layer pairs, where each pair appears in exactly one set $T_{y}$. Moreover, in graph $\mathcal{L}^{\prime}$, there is an edge from $s_{i}(y)$ to every vertex $u(y, h)$ with $(y, h) \in T_{y}$, and every such vertex connects to $t_{i}\left(y^{\prime}\right)$, which is the sink corresponding to source $s_{i}(y)$, i.e., $\left(s_{i}(y), t_{i}\left(y^{\prime}\right)\right) \in \mathcal{M}^{*}$. Additionally, for each pair of vertices $u\left(y^{\prime}, h^{\prime}\right), u\left(y^{\prime \prime}, h^{\prime \prime}\right)$ with $\left(y^{\prime}, h^{\prime}\right),\left(y^{\prime \prime}, h^{\prime \prime}\right) \in T_{y}$ and $h^{\prime}<h^{\prime \prime}$, there is an edge from $u\left(y^{\prime}, h^{\prime}\right)$ to $u\left(y^{\prime \prime}, h^{\prime \prime}\right)$ in $\mathcal{L}^{\prime}$.

Let $Y^{\prime} \subseteq Y$ denote the subset of labels $y$, for which the number of label-layer pairs $\left(y_{h}, h\right) \in T_{y}$ such that $i$ is not covered at $\left(y_{h}, h\right)$ is at least $L$. Since the total number of label-layer pairs $\left(y_{h}, h\right)$ for which $i$ is not covered at $\left(y_{h}, h\right)$ is at least $4|Y| L$, we have

$$
\left|Y^{\prime}\right|(32 L)+\left(|Y|-\left|Y^{\prime}\right|\right) L \geq 4|Y| L .
$$

It follows that $\left|Y^{\prime}\right| \geq(3 / 31)|Y|$. Now fix any label $y \in Y^{\prime}$. We will show that the source-sink pair $s_{i}(y)-t_{i}\left(y^{\prime}\right)$, where $y^{\prime}=y \oplus\left((32 L+1) \mu_{i}\right)$ is not disconnected when $\mathcal{S}$ is removed from the graph. Since there are $|Y|$ source-sink pairs of type $i$, it follows that set $\mathcal{S}$ does not disconnect at least $3 / 31$-fraction of these pairs, which will complete the proof.

For a fixed $y \in Y^{\prime}$, let $1 \leq h_{1}<h_{2}<\cdots<h_{L}<32 L$ be indices of layers, such that for each $j: 1 \leq j \leq L, i$ is not covered at the label-layer pair $\left(y_{h_{j}}, h_{j}\right) \in T_{y}$, where $y_{h_{j}}=y \oplus\left(h_{j} \mu_{i}\right)$. For each such $j: 1 \leq j \leq L$, we know that $S_{j}(r) \not \mathcal{S}_{y_{h_{j}}, h_{j}}$. In particular, there is some $p_{j} \in S_{j}$, such that vertex $v\left(y_{h_{j}}, h_{j}, p_{j}\right)$ does not belong to $\mathcal{S}$. We construct a path $P$ connecting source $s_{i}(y)$ to its sink $t_{i}(y)$, that contains type- $i$ edges only, as follows:

$$
P=\left(s_{i}(y) \rightarrow v\left(y_{h_{1}}, h_{1}, p_{1}\right) \rightarrow \cdots \rightarrow v\left(y_{h_{L}}, h_{L}, p_{L}\right) \rightarrow t_{i}\left(y^{\prime}\right)\right)
$$

¿From the discussion above, the non-terminal vertices appearing on this path do not belong to $\mathcal{S}$. We only need to check that indeed for every pair of consecutive vertices on the path there is a type- $i$ edge connecting them in the graph $G$. This is immediate from the definition of type- $i$ edges in graphs $H$ and $\mathcal{L}^{\prime}$.

Since there is a fractional solution of cost $N / L$ for the graph $G$, we have that the integrality gap is $\Omega(L)=\Omega\left(\frac{N^{1 / 7}}{\log ^{4 / 7} N}\right)$. Moreover, this gap holds even when the integral solution needs to disconnect only a $(1-\epsilon)$-fraction of the pairs where $\epsilon \geq 1 / 100$.

We have thus established the following theorem. 
Theorem 3.1 The flow-cut gap between the maximum multicommodity flow and minimum multicut in directed graphs is $\tilde{\Omega}\left(N^{1 / 7}\right)$. Moreover, this gap holds on directed acyclic graphs and even when the integral solution is required to separate only a $(1-\epsilon)$-fraction of the pairs for some $\epsilon>0$.

\subsection{Flow-Cut Gap for Sparsest Cut}

We now build on the preceding result to show that a similar gap result holds for concurrent flow and sparsest cut even in directed acyclic graphs. We apply the transformation outlined in Section 2 to the graph $G$ constructed in the previous section to obtain an instance $G^{\prime}$ of the (edge version of) the directed multicut. Let $E^{0}$ denote the set of special edges in $G^{\prime}$ and let $K=n|Y|$ denote the number of source-sink pairs in $G^{\prime}$. Consider the following fractional solution to (LP2-P). For each source-sink pair $\left(s_{i}, t_{i}\right)$, we set $h_{i}^{\prime}=1 / K$. For each special edge $e \in E^{0}$, we set $x_{e}^{\prime}=1 / K L$. This is a feasible solution to (LP2-P) of cost $N / K L$.

Assume that the integrality gap of (LP2-P) is less than $g(N)$ for some function $g$. Using an argument similar to the one given in [11] for converting bicriteria hardness of undirected multicut to hardness of sparsest cut, we show that there is a subset $E^{1}$ of edges in graph $G^{\prime},\left|E^{1}\right|=O(N / L) g(N)$, whose removal disconnects 0.99 -fraction of source-sink pairs. We perform several iterations, while in each iteration we remove some edges from $G^{\prime}$ and disconnect some source-sink pairs. The iterations are performed while the number of source-sink pairs disconnected is less than $0.99 \mathrm{~K}$. It is easy to see that at the beginning of each iteration there is a fractional solution to (LP2-P) of cost $100 N / K L$ : each special edge $e \in E^{0}$ that belongs to the graph at the beginning of the current iteration is assigned $x_{e}^{\prime}=100 / K L$, and each source-sink pair which is still not disconnected (there are at least $K / 100$ of them) is assigned $h_{i}^{\prime}=100 / K$. This is a feasible solution to (LP2-P) of $\operatorname{cost} \varphi=100 \mathrm{~N} / K L$. Therefore, in each iteration, there is an (integral) cut $S$ of containing at most $\varphi g(N) k^{\prime}$ edges that separates $k^{\prime}$ pairs for some integer $k^{\prime} \geq 1$. We delete all edges in $S$ from $G^{\prime}$ as well as remove the pairs separated by $S$. We repeat this until the number of remaining source-sink pairs falls below $K / 100$. Summing up over all iterations, we obtain a set of at most $\varphi g(N) K=(100 N / L) g(N)=O((N / L) g(N))$ that separates at least $0.99 K$ pairs. The following theorem now easily follows from the above discussion and Theorem 3.1.

Theorem 3.2 The gap between concurrent multicommodity flow and (non-bipartite) sparsest cut is $\tilde{\Omega}\left(N^{1 / 7}\right)$.

\section{Hardness of Approximation for Directed Cut Problems}

In this section, we will establish Theorems 1.2 and 1.3. As in the case of the flow-cut gaps, we will start by establishing the hardness result for the (vertex) directed multicut problem. We will in fact show a somewhat stronger result, namely, hardness for a "bi-criteria" version of multicut where the approximation algorithm is required to disconnect only $(1-\epsilon)$-fraction of source-sink pairs for some specified $\epsilon>0$. As before, a simple argument can then be used to translate this "bi-criteria" flow-cut gap result to a similar flow-cut gap result for directed sparsest cut.

\subsection{Overview of the Directed Multicut Hardness}

We show a reduction from a general class of constraint satisfaction problem (CSP). In a constraint satisfaction problem we are given a set $X$ of variables defined over some domain $F$, and a set of constraints $\Psi$. Each constraint $\psi \in \Psi$ involves $D$ variables, and a list $R_{\psi}$ of assignment to variables of $\psi$ that satisfy this constraint is given as problem input. The goal is to find assignments to variables so as to satisfy maximum possible number of constraints. (A formal definition appears below). 
There is a natural way to reduce the constraint satisfaction problem to directed multicut using the techniques developed for the integrality gap construction. The main difference is in the way graph $H$ is constructed. The non-terminal vertices of $H$ will represent the variables and their assignments, i.e., for every variable $x \in X$ and for every assignment $a \in F$ to $x$, there is a non-terminal vertex $v(x, a)$ in graph $H$. The source-sink pairs will represent the constraints, namely, for each $\psi \in \Psi$, there is a source-sink pair $s_{\psi}-t_{\psi}$. For each $\psi \in \Psi$, we construct a set $E_{\psi}$ of edges, by defining subsets $S_{j}(\psi)$ of vertices (layers), which are connected to each other and to the source-sink pairs as before. The subsets $S_{j}(\psi)$ will correspond to the satisfying assignments in $R_{\psi}$. Thus, if $A_{j}$ is the $j$ th satisfying assignment in $R_{\psi}$, then $S_{j}(\psi)$ will contain, for each variable $x$ participating in $\psi$, the vertex $v(x, a)$, where $a$ is the projection of $A_{j}$ onto $x$. The rest of the construction, including the labeling scheme $\mathcal{L}$, its transformed version $\mathcal{L}^{\prime}$ and the composition of $\mathcal{L}^{\prime}$ with $H$ remain the same. Unfortunately, this approach does not work with general constraint satisfaction problems. The main difficulty is that the same vertex $v(x, a)$ might belong to several sets $S_{j}(\psi)$ (for some fixed $\psi$ ), and because of this we cannot ensure that in the case of YES-INSTANCE the "standard" solution will disconnect all the source-sink pairs. Alternatively, if we view the constructed graph as an integrality gap example, we will have some short canonical paths, and hence we will not obtain large gap. As noted in the introduction, this problem does not arise in the CSPs obtained from the Raz verifier (where $D=2$ ), due to the projection property. However, the strongest possible hardness achievable via this approach is $2^{\Omega\left(\log ^{1-\epsilon} n\right)}$. This is a consequence of the fact that the hardness of our starting point, namely, the constraint satisfaction problem defined by the Raz verifier, itself is only $2^{\Omega\left(\log ^{1-\epsilon} n\right)}$. To break this barrier, we need to allow reductions from more general type of CSPs that do not necessarily have an analog of the projection property. We give here an overview of our approach to handle general CSPs.

To overcome the difficulty that the same vertex $v(x, a)$ might belong to several sets $S_{j}(\psi)$ for some fixed $\psi$, we create many copies of each vertex $v(x, a)$ representing assignment $a$ to variable $x$. Now layers $S_{j}(\psi)$ will use different copies for different indices $j$, thus avoiding the creation of these bad paths. However, we need to enforce consistency among multiple copies of an assignment to a variable. Specifically, we would like to ensure the following. Fix any solution $\mathcal{S}$, and say that a variable-assignment pair $(x, a)$ is chosen by $\mathcal{S}$ iff at least $1 / 4$ of the copies of corresponding vertices belong to $\mathcal{S}$. Let $\psi$ be any constraint for which no satisfying assignment is chosen by $\mathcal{S}$. We want to ensure that in this case there is an $s$ - $t$ pair corresponding to $\psi$, which is not disconnected by $\mathcal{S}$. To achieve this goal, instead of choosing the layers $S_{1}(\psi), \ldots, S_{L}(\psi)$ just once, we perform $|Y| \Gamma$ such independent choices, each one of them defining a different subset $E_{y, \gamma}$ of edges in graph $H$, for $y \in Y, 1 \leq \gamma \leq \Gamma$ (here $\Gamma \leq \operatorname{poly}(n)$ and $Y$ is the set of labels). For a fixed $y \in Y, \gamma: 1 \leq \gamma \leq \Gamma$, in order to choose a subset $S_{j}(\psi)$ of vertices, we randomly choose $\lambda=O(\log n)$ copies of every vertex representing the variable-assignment pair $(x, a)$ where $x$ is a variable of $\psi$ and the projection of the $j$ th assignment in $R_{\psi}$ onto $x$ is $a$. The random choices are performed with no repetitions across various layers, so each copy may appear in at most one layer for a fixed pair $y, \gamma$ and a fixed constraint $\psi$. Let $g(n)$ denote the hardness gap of the constraint satisfaction system, where $n$ is the number of variables in the system. The resulting instance has the property that with high probability, the cost of any No-instance solution is at least $g(N)^{\delta}$ times the cost of an optimal Yesinstance solution even when the No-instance solution is only required to separate a $\left(1-\epsilon^{*}\right)$-fraction of source-sink pairs for some constant $\epsilon^{*}>0$ (here $N$ is the size of the resulting instance, and $\delta>0$ is some constant). Using standard arguments, we can then translate the hardness for this bicriteria version to a matching hardness for sparsest cut in directed graphs. 


\subsection{Starting Point: The Generalized Label Cover Problem}

We perform a reduction from a variation of the label cover problem, that we refer to as the generalized label cover, based on the notion of constraint satisfaction systems.

Definition: A constraint satisfaction system $\mathcal{C}=(\Psi, X, F, R, D)$ consists of a set $X$ of variables defined over an alphabet $F$ and a set $\Psi$ of constraints, depending on $D$ variables each. For each constraint $\psi \in \Psi$, a set $R_{\psi} \subseteq F^{D}$ of satisfying assignments to this constraint is given. Let $|X|=n$ and $|\Psi|=m$. We say $x \in \psi$ to indicate that the constraint $\psi$ depends on variable $x$. For each constraint $\psi \in \Psi$, let $k_{\psi}=\left|R_{\psi}\right|$ be the number of assignments to variables of $\psi$ that satisfy this constraint.

We use the following theorem which provides a low-error PCP.

Theorem 4.1 [18] For every constant $\epsilon_{0}>0$, there exist constants $c_{1}, c_{2}>0$, such that for constraint satisfaction systems $\mathcal{C}=(\Psi, X, F, R, D)$, where $D=c_{1}$ and $|F|=2^{(\log m)^{1-\epsilon_{0}}}$, it is $N P$-hard to distinguish between the two following cases:

- Yes-Instance: There is an assignment to the variables that satisfies all the constraints.

- No-Instance: No assignment can satisfy more than a $c_{2} /|F|$ fraction of the constraints.

In the generalized label cover problem we are given a constraint satisfaction system $\mathcal{C}=(\Psi, X, F, R, D)$, and additionally, for each variable $x \in X$, a positive integer weight $w_{x}$ is specified. Let $W=$ $\sum_{x \in X} w_{x}$. A solution is a function $f: X \rightarrow 2^{F}$, that assigns each variable $x \in X$ a subset of values $f(x) \subseteq F$. Solution cost is the total weighted sum of the number of assignments chosen, i.e., $\sum_{x \in X} w_{x}|f(x)|$. We say that a constraint $\psi \in \Psi$ is satisfied by the solution $f$, iff there exists a satisfying assignment $A \in R_{\psi}$, such that for each variable $x$ participating in $\psi$, the value of $A$ projected onto $x, A_{\mid x}$, belongs to $f(x)$. In other words, if $x_{j_{1}}, \ldots, x_{j_{D}}$ are the variables of $\psi$, and if $a_{1}, \ldots, a_{D}$ is the projection of $A$ onto these variables respectively, then for each $h: 1 \leq h \leq D$, $a_{h} \in f\left(x_{j_{h}}\right)$. The goal is to find a minimum-cost solution that satisfies all the constraints.

The starting point of our reduction is the next theorem:

Theorem 4.2 For any constant $\epsilon>0$, it is NP-hard to distinguish between the following two cases of the label cover problem, even when $W,|F|^{D}, n \leq \operatorname{poly}(m)$ :

- Yes-Instance: there is a solution for the label cover problem of cost $W$.

- No-Instance: any solution of cost less than $g W$ satisfies less than $\frac{1}{2}$ of the constraints, for $g=2^{(\log m)^{1-\epsilon}}$.

Proof: Let $\mathcal{C}=(\Psi, X, F, D)$ be the constraint satisfaction system from Theorem 4.1. For each variable $x \in X$ we define its weight $w_{x}$ to be the number of constraints in $\Psi$ that involve $x$.

Notice that if $\mathcal{C}$ is a YeS-Instance, then there is a solution to the label cover problem of cost $W$, which is defined by the satisfying assignment to the constraint satisfaction system $\mathcal{C}$, where each variable is assigned one value in $F$.

Assume now that $\mathcal{C}$ is a No-Instance. Let $f$ be any solution to the corresponding label cover problem, whose cost is less than $g W$. We show that less than $\frac{1}{2}$-fraction of the constraints are satisfied by $f$. 
We say that variable $x$ is bad iff $|f(x)| \geq 4 D g$. We say that a constraint $\psi \in \Psi$ is bad iff it contains at least one bad variable. We show that at most $1 / 4$ of the constraints are bad and that less than $m / 4$ good constraints are satisfied.

To bound the number of bad constraints, recall that for each variable $x \in X, w_{x}$ is the number of constraints in which $x$ appears. Therefore the solution cost can be equivalently written as $\sum_{\psi \in \Psi} \sum_{x \in \psi}|f(x)|$. Therefore, if $\mathcal{B}$ denotes the set of bad constraints, then the solution cost is at least $|\mathcal{B}| \cdot 4 D g$. Assume that more than $1 / 4$ of the constraints are bad. Then the solution cost is greater than $\frac{m}{4} \cdot 4 D g \geq g W$ (since $W \leq D m$ ), which is a contradiction. Therefore, the fraction of bad constraints is at most $1 / 4$.

We now prove that the number of good constraints which are satisfied by the assignment is less than $m / 4$. Assume otherwise. We show an assignment to variables of $X$ that satisfies many constraints. For each good variable $x \in X$ we randomly choose one of its at most $4 D g$ assignments from $f(x)$. The expected number of constraints thus satisfied is at least $\frac{m}{4(4 D g)^{D}}$. Choosing $g=\Theta\left(\frac{|F|^{1 / D}}{D}\right)$ completes the proof of the theorem, by noticing that $g \geq 2^{(\log m)^{1-\epsilon}}$, where $\epsilon$ can be any constant (by appropriately setting the constant $\epsilon_{0}$ in Theorem 4.1). Notice also that $W=\operatorname{poly}(m)$ and $n,|F|^{D} \leq \operatorname{poly}(m)$.

\subsection{Reduction from the Generalized Label Cover Problem to Directed Multicut}

We will reduce the generalized label cover problem to the weighted vertex version of directed multicut. In this problem every non-terminal vertex $v$ has a positive integer weight $w_{v}$ associated with it, and the goal is to find a minimum-weight subset of vertices whose removal disconnects all the source-sink pairs. The weights $w_{v}$ that we use are polynomial in the problem instance size. It is easy to transform such weighted instances to unweighted instances where minimum multicut has the same cost: every non-terminal vertex $v$ is replaced by $w_{v}$ copies. For every edge $(u \rightarrow v)$ in the original graph, we add an edge from every copy of $u$ to every copy of $v$. It is easy to see that optimal solution costs in both graphs are the same, and instance size grows by polynomial factor only. Hence, our hardness of approximation results hold for the unweighted vertex version, and following the reduction outlined in Section 2, for the edge version of multicut as well.

The reduction uses the ideas from the integrality gap construction. In particular, we define an initial graph $H$ and a transformed labeling scheme $\mathcal{L}^{\prime}$. The composition of these two graphs produces our final graph $G$. Since the transformed labeling scheme is similar to the one appearing in the integrality gap construction, we describe it in the first step. In the second step, we construct graph $H$, which will play the role of the initial graph $H$ in the integrality gap construction. In the third and the final step we combine the two graphs to obtain the final graph $G$.

\subsubsection{Step 1: The Labeling Scheme}

Let $\Gamma=2^{20} D|F|^{2 D+1} \leq$ poly $(n)$ be a parameter. We will use the transformed labeling scheme $\mathcal{L}^{\prime}=\left(U, \mathcal{M}, E^{\prime}\right)$ from the integrality gap construction with parameters $\tau=\Gamma m$ and $Z=64|F|^{D}$. Recall that the set of labels is $Y=[2 \tau Z] \times\left[2 \tau^{2} Z\right]$. We also have a collection of $\Gamma m$ increment vectors, which we denote by $\mu_{\psi, \gamma}$ for $\psi \in \Psi$ and $1 \leq \gamma \leq \Gamma$. For the sake of completeness we briefly describe $\mathcal{L}^{\prime}$ here (there is also a slight change of notation).

- Non-terminal vertices: $U=\{u(y, h) \mid y \in Y, 1 \leq h \leq Z\}$.

- Source-sink pairs: $\mathcal{M}=\left\{\left(s_{\psi, \gamma}(y), t_{\psi, \gamma}\left(y^{\prime}\right)\right) \mid y \in Y, \psi \in \Psi, 1 \leq \gamma \leq \Gamma, y^{\prime}=y \oplus\left((Z+1) \mu_{\psi, \gamma}\right)\right\}$.

- Edges: For each $\psi \in \Psi$ and for each $\gamma: 1 \leq \gamma \leq \Gamma$, we define a set $E_{\psi, \gamma}^{\prime}$ of edges, and set 


$$
\begin{aligned}
& E^{\prime}=\bigcup_{\substack{\psi \in \Psi \leq \Gamma \\
1 \leq \gamma, \gamma}} E_{\psi}^{\prime} \text { The set } E_{\psi, \gamma}^{\prime} \text { of edges is defined as follows: } \\
& \qquad \begin{aligned}
E_{\psi, \gamma}^{\prime} & =\left\{u(y, h) \rightarrow u\left(y^{\prime}, h+c\right) \mid c>0, y^{\prime}=y \oplus\left(c \mu_{\psi, \gamma}\right)\right\} \\
& \cup\left\{s_{\psi, \gamma}(y) \rightarrow u\left(y^{\prime}, h\right) \mid y^{\prime}=y \oplus\left(h \mu_{\psi, \gamma}\right)\right\} \\
& \cup\left\{u\left(y^{\prime}, h\right) \rightarrow t_{\psi, \gamma}\left(y^{\prime \prime}\right) \mid y^{\prime \prime}=y^{\prime} \oplus\left((Z+1-h) \mu_{\psi, \gamma}\right)\right\}
\end{aligned}
\end{aligned}
$$

We will further partition the set $E_{\psi, \gamma}^{\prime}$ of edges into $|Y|$ subsets $E_{y, \psi, \gamma}^{\prime}$ for $y \in Y$ as follows: edge $e: u \rightarrow v \in E_{\psi, \gamma}^{\prime}$ belongs to set $E_{y, \psi, \gamma}^{\prime}$ iff there is an edge from $s_{\psi, \gamma}(y)$ to $u$ in $E_{\psi, \gamma}^{\prime}$ or $s_{\psi, \gamma}(y)=u$. The edges in set $E_{y, \psi, \gamma}^{\prime}$ are called the edges of type $(y, \psi, \gamma)$.

For any source-sink pair $s_{\psi, \gamma}(y)-t_{\psi, \gamma}\left(y^{\prime}\right)$, we say that a path $P$ connecting the source to the sink is canonical iff it only uses edges of type $(y, \psi, \gamma)$. Using an argument similar to one given in Claim 3.1, it is easy to show that there are no non-canonical source-sink paths in the graph $\mathcal{L}^{\prime}$.

\subsubsection{Step 2: Graph $H$}

We define graph $H=(V, \mathcal{M}, E)$ in this section.

Let $\lambda=O(\log n)$, the exact value to be fixed later. We first describe the set $V$ of non-terminal vertices. For each variable $x \in X$, for each assignment $a \in F$ to this variable, we create a set $V(x, a)$ of $C=4 \lambda|F|^{D}$ vertices, $V(x, a)=\{v(x, a, b) \mid 1 \leq b \leq C\}$. Vertices in set $V(x, a)$ represent assignment $a$ to variable $x$. Let $V(x)=\bigcup_{a \in F} V(x, a)$, and let $V=\bigcup_{x \in X} V(x)$ be the set of all the non-terminal vertices of graph $H$.

The source-sink pairs are defined exactly as in graph $\mathcal{L}^{\prime}$, i.e.,

$$
\mathcal{M}=\left\{\left(s_{\psi, \gamma}(y), t_{\psi, \gamma}\left(y^{\prime}\right)\right) \mid y \in Y, \psi \in \Psi, 1 \leq \gamma \leq \Gamma, y^{\prime}=y \oplus\left((Z+1) \mu_{\psi, \gamma}\right)\right\} .
$$

Finally, we need to define the set of edges. For each $\psi \in \Psi, \gamma: 1 \leq \gamma \leq \Gamma$ and for each $y \in Y$, we define a set $E_{y, \psi, \gamma}$ of edges of type $(y, \psi, \gamma)$. The final set of edges of graph $H$ is $E=\bigcup_{\substack{y \in Y, \psi \in \Psi \\ 1 \leq \gamma \leq \Gamma}} E_{y, \psi, \gamma}$.

Fix some constraint $\psi \in \Psi$, label $y \in Y$ and index $\gamma: 1 \leq \gamma \leq \Gamma$. We show how to define the set $E_{y, \psi, \gamma}$ of edges. Let $\left|R_{\psi}\right|=k_{\psi}$, and let $x_{i_{1}}, \ldots, x_{i_{D}}$ be the variables participating in constraint $\psi$. We construct $k_{\psi}$ disjoint sets of non-terminal vertices $S_{1}(y, \psi, \gamma), \ldots, S_{k_{\psi}}(y, \psi, \gamma)$. The set $E_{y, \psi, \gamma}$ of edges will contain an edge from $s_{\psi, \gamma}(y)$ to every vertex of $S_{1}(y, \psi, \gamma)$, an edge from every vertex of $S_{k_{\psi}}(y, \psi, \gamma)$ to $t_{\psi, \gamma}(y)$, and additionally for each $j: 1 \leq j<k_{\psi}$, an edge from every vertex of $S_{j}(y, \psi, \gamma)$ to every vertex of $S_{j+1}(y, \psi, \gamma)$.

Finally, we need to specify how subsets $S_{j}(y, \psi, \gamma)$ are determined. We assume that the assignments in $R_{\psi}$ are ordered in some arbitrary way. Let $A_{j}$ be the $j$ th assignment in $R_{\psi}$. Let $A_{j}=\left(a_{1}, \ldots, a_{D}\right)$ be projections of $A_{j}$ onto the variables $x_{i_{1}}, \ldots, x_{i_{D}}$, respectively. For each $\ell: 1 \leq \ell \leq D$, let $B_{\ell} \subseteq V\left(x_{i_{\ell}}, a_{\ell}\right)$ be the set of all the vertices representing assignment $a_{\ell}$ to variable $x_{i_{\ell}}$, which have not been used in sets $S_{1}(y, \psi, \gamma), \ldots, S_{j-1}(y, \psi, \gamma)$. We randomly choose $\lambda$ vertices from $B_{\ell}$ and add them to set $S_{j}(y, \psi, \gamma)$. We do this for every $\ell: 1 \leq \ell \leq D$. Since $C=4 \lambda|F|^{D}$ and $k_{\psi} \leq|F|^{D}$, this is possible to do.

\subsubsection{Step 3: The Final Construction}

In this step we create our final graph $G=\left(V^{\prime}, \mathcal{M}, E^{*}, w\right)$ by combining graphs $\mathcal{L}^{\prime}=\left(U, \mathcal{M}, E^{\prime}\right)$ and $H=(V, \mathcal{M}, E)$.

The set of non-terminal vertices of graph $G$ is $V^{\prime}=V \times U$, i.e.,

$$
V^{\prime}=\{v(y, h, x, a, b) \mid y \in Y, 1 \leq h \leq Z, x \in X, a \in F, 1 \leq b \leq C\}
$$


For each non-terminal vertex $v(y, h, x, a, b)$, we define its pre-image in graph $H$ to be $g_{H}(v(y, h, x, a, b))=$ $v(x, a, b)$ and its pre-image in graph $\mathcal{L}^{\prime}$ to be $g_{\mathcal{L}^{\prime}}(v(y, h, x, a, b))=u(y, h)$. The weight of the nonterminal vertex $v(y, h, x, a, b)$ is $w_{x}$ (the weight of variable $x$ in the label-cover problem).

The set of source-sink pairs is exactly the same as in $H$ and $\mathcal{L}^{\prime}$. Thus,

$$
\mathcal{M}=\left\{\left(s_{\psi, \gamma}(y), t_{\psi, \gamma}\left(y^{\prime}\right)\right) \mid y \in Y, \psi \in \Psi, 1 \leq \gamma \leq \Gamma, y^{\prime}=y \oplus\left((Z+1) \mu_{\psi, \gamma}\right)\right\} .
$$

For each source $s_{\psi, \gamma}$, its pre-image in graph $H$ is defined to be $g_{H}\left(s_{\psi, \gamma}\right)=s_{\psi, \gamma}$ and its pre-image in graph $\mathcal{L}^{\prime}$ is defined to be $g_{\mathcal{L}^{\prime}}\left(s_{\psi, \gamma}\right)=s_{\psi, \gamma}$. The pre-images of sinks are defined similarly.

Finally, we need to define the set of edges. For each $y \in Y, \psi \in \Psi, 1 \leq \gamma \leq \Gamma$, we define a set $E_{y, \psi, \gamma}^{*}$ of edges of type $(y, \psi, \gamma)$ and we set $E^{*}=\bigcup_{\substack{y \in Y, \psi \in \Psi \\ 1 \leq \gamma \leq \Gamma}} E_{y, \psi, \gamma}^{*}$.

For any pair $(u, v)$ of vertices (terminal or non-terminal), we add an edge of type $(y, \psi, \gamma)$ from $u$ and $v$ iff there is an edge of type $(y, \psi, \gamma)$ from $g_{H}(u)$ to $g_{H}(v)$ in graph $H$ and there is an edge of type $(y, \psi, \gamma)$ from $g_{\mathcal{L}^{\prime}}(u)$ to $g_{\mathcal{L}^{\prime}}(v)$ in $\mathcal{L}^{\prime}$.

This concludes the description of our final construction. We now discuss its properties.

Construction Size: The construction size $N$ is bounded by:

$$
\begin{aligned}
N & =|Y| Z|X||F| C=O\left(\tau^{3} Z^{2}\right) \cdot 64|F|^{D} \cdot n \cdot|F| \cdot 4 \lambda|F|^{D} \\
& \leq O\left(\Gamma^{3} m^{3}|F|^{3 D} \cdot n \cdot|F|^{D+1} \cdot \lambda\right) \\
& \leq O\left(D^{3}|F|^{6 D+3} m^{3}|F|^{3 D} \cdot n \cdot|F|^{D+1} \cdot \lambda\right) \\
& \leq O\left(D^{3}|F|^{10 D+4} m^{3} n \log n\right) \\
& =\operatorname{poly}(m, n) \\
& \leq \operatorname{poly}(m)
\end{aligned}
$$

Note that it follows that a gap of $2^{\left(\log ^{1-\epsilon} m\right)}$ can be expressed as $2^{\Omega\left(\log ^{1-\epsilon} N\right)}$.

\subsubsection{The Hardness Gap Analysis}

We next analyze the gap between the cost of a Yes-Instance solution and a No-Instance solution.

\section{Yes-Instance Cost:}

We say a path connecting source $s_{\psi, \gamma}(y)$ to $\operatorname{sink} t_{\psi, \gamma}(y)$ is a canonical path iff it only uses edges of type $(y, \psi, \gamma)$. Consider a solution to the label-cover problem instance of cost $W$. In this solution, for each variable $x \in X$, exactly one assignment $\alpha(x) \in F$ is chosen, and the chosen assignments satisfy all the constraints. Let $\mathcal{S}$ be a set of vertices, containing, for every $x \in X$, the subset $\{v(y, h, x, \alpha(x), b) \mid y \in Y, 1 \leq h \leq Z, 1 \leq b \leq C\}$ of vertices. The cost of this solution is:

$$
\mathcal{C}_{Y I}=|Y| Z W C
$$

We next prove that $\mathcal{S}$ is a feasible solution. The proof consists of two parts. First, we prove that no non-canonical paths exist in the graph. After that we prove that $\mathcal{S}$ disconnects all the canonical paths.

Lemma 4.1 For every source-sink pair $s_{\psi, \gamma}(y)-t_{\psi, \gamma}(y)$, the only paths in $G$ connecting the source to the sink are the canonical paths. 
Proof: Assume otherwise, and let $s_{\psi, \gamma}(y)-t_{\psi, \gamma}(y)$ be any source-sink pair for which a non-canonical path $\mathcal{P}$ exists in the graph $G$. Let $\mathcal{P}^{\prime}$ be a sequence of vertices in graph $\mathcal{L}^{\prime}$ containing the pre-images of vertices on path $\mathcal{P}$ in the exact order in which they appear on this path. Because of the way the edges of $G$ are defined, $\mathcal{P}^{\prime}$ must be a non-canonical path connecting $s_{\psi, \gamma}(y)$ to $t_{\psi, \gamma}(y)$ in graph $\mathcal{L}^{\prime}$, which is impossible.

Lemma 4.2 All the canonical source-sink paths in $G$ are disconnected when $\mathcal{S}$ is removed from the graph.

Proof: Assume otherwise. Let $P$ be a canonical path connecting some source-sink pair $s_{\psi, \gamma}(y)$ $t_{\psi, \gamma}(y)$ in $G$ whose vertices do not belong to $\mathcal{S}$. Let $P^{\prime}$ be a sequence of vertices in graph $H$ containing the pre-images of vertices on path $P$ in the exact order in which they appear on $P$. Then $P^{\prime}$ must be a canonical path connecting $s_{\psi, \gamma}(y)$ to $t_{\psi, \gamma}(y)$ in graph $H$. Since $P^{\prime}$ uses edges of type $(y, \psi, \gamma)$ only, we know that for every $j: 1 \leq j \leq k_{\psi}$, the $(j+1)$ st vertex on path $P^{\prime}$ belongs to $S_{j}(y, \psi, \gamma)$. Since the solution to the label cover problem satisfies all the constraints, there must be an assignment $A_{j^{*}} \in R_{\psi}, 1 \leq j^{*} \leq k_{\psi}$ such that for each $x \in \psi$, the projection of $A_{j^{*}}$ onto $x$ is $\alpha(x)$. But then set $\mathcal{S}$ contains all the vertices in the set $\left\{v(y, h, x, a, b) \mid y \in Y, 1 \leq h \leq Z, v(x, a, b) \in S_{j^{*}}(y, \psi, \gamma)\right\}$ and at least one vertex from this set must lie on $P$.

\section{No-Instance Cost:}

Consider any subset $\mathcal{S} \subseteq V^{\prime}$ of vertices whose total weight is less than $g C_{Y I} / 8$, where $g=$ $2^{\left(\log ^{1-\epsilon} m\right)}=2^{\Omega\left(\log ^{1-\epsilon} N\right)}$ is the parameter from Theorem 4.2 .

Fix any label-layer pair $(y, h), y \in Y, 1 \leq h \leq Z$. For each variable $x \in X$, we define a set $\mathcal{S}_{y, h}(x) \subseteq$ $F$ of assignments $a$, such that at least $1 / 4$-fraction of the vertices $\{v(y, h, x, a, b) \mid 1 \leq b \leq C\}$ belong to set $\mathcal{S}$. We view the assignments in $\mathcal{S}_{y, h}(x)$ as if they are chosen by the solution $\mathcal{S}$ for variable $x$ at label-layer pair $(y, h)$.

We now define a set $\Psi_{y, h} \subseteq \Psi$ of constraints, that are covered by solution $\mathcal{S}$ at label-layer pair $(y, h)$. Constraint $\psi$ belongs to $\Psi_{y, h}$ iff there is a satisfying assignment $A \in R_{\psi}$, such that for each variable $x \in \psi$, assignment $A_{\mid x}$ is chosen by $\mathcal{S}$ at $(y, h)$, or in other words $A_{\mid x} \in \mathcal{S}_{y, h}(x)$.

We now proceed as follows. Using simple counting arguments we show that for most label-layer pairs $(y, h)$, the size of set $\Psi_{y, h}$ is small, and thus most constraints are not covered by $\mathcal{S}$ at $(y, h)$. We will then argue that for a constant fraction of constraints $\psi \in \Psi$, the number of label-layer pairs $(y, h)$ in which $\psi$ is not covered is large. Finally, we use the probabilistic method to show that with high probability, a constant fraction of source-sink pairs corresponding to such constraints $\psi$ are not disconnected when $\mathcal{S}$ is removed from the graph (and this happens due to the random choices in constructions of the subsets $S_{j}(y, \psi, \gamma)$ of vertices in $H$.)

We now proceed with the formal proof.

A constraint $\psi$ is called good iff the number of label-layer pairs $(y, h)$ for which $\psi \notin \Psi_{y, h}$ is at least $|Y| Z / 8$. Let $\mathcal{G} \subseteq \Psi$ denote the subset of good constraints.

Claim 4.1 $|\mathcal{G}| \geq m / 8$.

Proof: Fix some label-layer pair $(y, h)$, where $y \in Y, 1 \leq h \leq Z$. Recall that for each variable $x \in X$, we have defined a subset of assignments $\mathcal{S}_{y, h}(x)$. Consider a solution to the label-cover problem, where $f(x)=\mathcal{S}_{y, h}(x)$. Let $W_{y, h}$ denote the cost of this solution. We say that $(y, h)$ is a good label-layer pair, iff $W_{y, h}<g W$. 
We claim that at least half of the label-layer pairs are good. Otherwise, we have more than $|Y| Z / 2$ non-good label-layer pairs $(y, h)$, with $W_{y, h} \geq g W$. Recall that for each variable $x \in X$, if assignment $a \in \mathcal{S}_{y, h}(x)$, then $\mathcal{S}$ contains at least $C / 4$ of the vertices $\{v(y, h, x, a, b) \mid 1 \leq b \leq C\}$. Therefore, the cost of $\mathcal{S}$ must be more than $\frac{|Y| Z}{2} \frac{C}{4} \cdot g W \geq g \cdot \frac{|Y| Z C W}{8}=\frac{g}{8} C_{Y I}$, which is a contradiction.

Following Theorem 4.2, each one of the good label-layer pairs covers less than half the constraints, i.e., if $(y, h)$ is good then $\left|\Psi_{y, h}\right|<m / 2$.

Let $T$ be a set of triples $(y, h, \psi)$, where $(y, h)$ is a good label-layer pair that does not cover constraint $\psi$, i.e., $\psi \notin \Psi_{y, h}$. From the above discussion, $|T| \geq \frac{|Y| Z}{2} \cdot \frac{m}{2}=|Y| Z m / 4$.

Assume now for contradiction that $|\mathcal{G}|<m / 8$. Then each constraint in $\mathcal{G}$ contributes at most $|Y| Z$ triples to set $T$, and there are less than $m / 8$ such constraints. Constraints $\psi \notin \mathcal{G}$ contribute at most $|Y| Z / 8$ triples each and there are at most $m$ such constraints. Therefore in total $|T|<|Y| Z m / 4$ must hold, which is a contradiction.

We now fix some good constraint $\psi \in \mathcal{G}$, and our goal is to show that at least $1 / 32$-fraction of the source-sink pairs $s(y, \psi, \gamma)-t(y, \psi, \gamma)$ for $y \in Y, 1 \leq \gamma \leq \Gamma$ are not disconnected when $\mathcal{S}$ is removed from $G$, with high probability.

Let $V_{x}=\{v(y, h, x, a, b) \mid y \in Y, 1 \leq h \leq Z, a \in F, 1 \leq b \leq C\}$ be the set of non-terminal vertices representing variable $x$, and let $V_{\psi}=\bigcup_{x \in \psi} V(x)$. Consider any subset $\mathcal{S}^{\prime} \subseteq V_{\psi}$ of vertices, such that the number of label-layer pairs $(y, h)$ for which $\psi \notin \Psi_{y, h}$ is at least $|Y| Z / 8$ (here $\Psi_{y, h}$ is defined with respect to $\mathcal{S}^{\prime}$ and not $\left.\mathcal{S}\right)$. For a constraint $\psi$, we say that:

- Bad event $\eta_{\mathcal{S}^{\prime}}(y, \psi, \gamma)$ happens if the removal of $\mathcal{S}^{\prime}$ from the graph $G$ disconnects $s_{\psi, \gamma}(y)$ from $t_{\psi, \gamma}(y)$.

- Bad event $\eta_{\mathcal{S}^{\prime}}(\psi)$ happens iff there are at least $31|Y| \Gamma / 32$ pairs $(y, \gamma)$ where $y \in Y, 1 \leq \gamma \leq \Gamma$ for which the bad event $\eta_{\mathcal{S}^{\prime}}(y, \psi, \gamma)$ happens.

- Bad event $\eta(\psi)$ happens iff there is at least one subset $\mathcal{S}^{\prime} \subseteq V_{\psi}$ as defined above such that $\eta_{\mathcal{S}^{\prime}}(\psi)$ is true.

Lemma 4.3 For any good constraint $\psi \in \mathcal{G}$, the probability of $\eta(\psi)$ is at most $1 / 2 m$.

Proof: Let $\mathcal{S}^{\prime} \subseteq V_{\psi}$ be as defined above. Let $\beta$ be the subset of label-layer pairs $(y, h)$, for which $\psi \notin \Psi_{y, h}$. Recall that $|\beta| \geq|Y| Z / 8$.

Now for any $\gamma: 1 \leq \gamma \leq \Gamma$, we can partition the set $Y \times\{1, \ldots, Z\}$ of all the label-layer pairs into $|Y|$ subsets. For each $y \in Y$, we define the subset $P_{\gamma, y}=\left\{\left(y_{h}, h\right) \mid y_{h}=y \oplus\left(h \mu_{\psi, \gamma}\right)\right\}$. Let $Y_{\gamma} \subseteq Y$ be the set of labels $y$, such that $\left|\beta \cap P_{\gamma, y}\right| \geq Z / 16$. Clearly, $\left|Y_{\gamma}\right| \geq|Y| / 16$ : otherwise, each $y \in Y_{\gamma}$ contributes at most $Z$ pairs to $\beta$, and each $y \notin Y_{\gamma}$ contributes less than $Z / 16$ pairs to $\beta$. Thus, in total $|\beta|<Z \cdot \frac{|Y|}{16}+|Y| \cdot \frac{Z}{16} \leq \frac{|Y| Z}{8}$, which is a contradiction.

Here onwards, we fix a $\gamma: 1 \leq \gamma \leq \Gamma$ and a $y \in Y_{\gamma}$. We will first show that the probability of event $\eta_{\mathcal{S}^{\prime}}(y, \psi, \gamma)$ is at most $2^{-\lambda / 2}$. Consider the sets $S_{1}(y, \psi, \gamma), \ldots, S_{k_{\psi}}(y, \psi, \gamma)$ that were chosen when graph $H$ was constructed. For brevity, we denote these sets by $S_{1}, \ldots, S_{k}$ respectively, where $k=k_{\psi}$. Let $1 \leq \ell_{1} \leq \cdots \leq \ell_{k} \leq Z$ be layers, such that for all $h: 1 \leq h \leq k$, the label-layer pair $\left(y_{h}, \ell_{h}\right)$ belongs to $\beta$, where $y_{h}=y \oplus\left(\ell_{h} \cdot \mu_{\psi, \gamma}\right)$. Since $y \in Y_{\gamma}$, there are at least $Z / 16>|F|^{D} \geq k$ such possible layers. Therefore, $\psi$ is not covered by $\mathcal{S}^{\prime}$ at $\left(y_{h}, \ell_{h}\right)$ for all $h: 1 \leq h \leq k$, and in particular if we consider the assignment $A_{h} \in R_{\psi}$, then there is at least one variable $x_{h} \in \psi$, such that if $a_{h}=\left(A_{h}\right)_{\mid x_{h}}$, then $a_{h} \notin \mathcal{S}_{y_{h}, \ell_{h}}\left(x_{h}\right)$, or in other words $\mathcal{S}^{\prime}$ contains less than $C / 4$ vertices from the set $T_{h}=\left\{v\left(y_{h}, \ell_{h}, x_{h}, a_{h}, b_{h}\right) \mid 1 \leq b_{h} \leq \mathcal{C}\right\}$. 
We define the following subset of vertices in graph $H$ :

$$
T_{h}^{\prime}=\left\{v\left(x_{h}, a_{h}, b_{h}\right) \mid v\left(y_{h}, \ell_{h}, x_{h}, a_{h}, b_{h}\right) \in \mathcal{S}^{\prime}\right\}
$$

It follow from the above discussion that $\left|T_{h}^{\prime}\right| \leq C / 4$. Recall that set $S_{h}$ contains $\lambda$ vertices from the set $\left\{v\left(x_{h}, a_{h}, b_{h}\right) \mid 1 \leq b_{h} \leq C\right\}$.

Assume first that $S_{h} \nsubseteq T_{h}^{\prime}$. Then there is a vertex $v\left(x_{h}, a_{h}, b_{h}\right) \in S_{h}$ such that $v\left(x_{h}, a_{h}, b_{h}\right) \notin T_{h}^{\prime}$ or in other words $v\left(y_{h}, \ell_{h}, x_{h}, a_{h}, b_{h}\right) \notin \mathcal{S}^{\prime}$. Therefore, if we have that for all $h: 1 \leq h \leq k, S_{h} \nsubseteq T_{h}^{\prime}$, then we have a sequence of vertices $v\left(y_{1}, \ell_{1}, x_{1}, a_{1}, b_{1}\right), \ldots, v\left(y_{k}, \ell_{k}, x_{k}, a_{k}, b_{k}\right)$ that do not belong to $\mathcal{S}^{\prime}$. Moreover, there is the following path in the graph $G$ :

$$
s_{\psi, \gamma}(y) \rightarrow v\left(y_{1}, \ell_{1}, x_{1}, a_{1}, b_{1}\right) \rightarrow \cdots \rightarrow v\left(y_{k}, \ell_{k}, x_{k}, a_{k}, b_{k}\right) \rightarrow t_{\psi, \gamma}\left(y^{\prime}\right)
$$

where $\left(s_{\psi, \gamma}(y), t_{\psi, \gamma}\left(y^{\prime}\right)\right)$ is a source-sink pair in $G$. (Notice that this path exists since for all $h: 1 \leq h \leq k$, the pre-image of $v\left(y_{h}, \ell_{h}, x_{h}, a_{h}, b_{h}\right)$ in graph $H$ belongs to $S_{h}$, and thus the preimages of the vertices on this path are connected by a canonical path in graph $H$. The same is true from graph $\mathcal{L}^{\prime}$.)

Therefore, if for all $h: 1 \leq h \leq k, S_{h} \nsubseteq T_{h}^{\prime}$, then the event $\eta_{\mathcal{S}^{\prime}}(y, \psi, \gamma)$ does not happen. The probability that $S_{h} \subseteq T_{h}^{\prime}$ for some fixed $h$, regardless of the choices made by previous layers (recall that the choices made by different layers are not independent) is at most $1 / 2^{\lambda}$. The probability that for some $h: 1 \leq h \leq k_{\psi}, S_{h} \subseteq T_{h}^{\prime}$ is bounded by $\frac{k_{\psi}}{2^{\lambda}} \leq \frac{|F|^{D}}{2^{\lambda}} \leq 2^{-\lambda / 2}$ for appropriate choice of $\lambda=O(\log n)$. Therefore, the probability of event $\eta_{\mathcal{S}^{\prime}}(y, \psi, \gamma)$ is at most $2^{-\lambda / 2}$.

Recall that the choices made for subsets $S_{j}(y, \psi, \gamma)$ for different values of $y \in Y, 1 \leq \gamma \leq \Gamma$ are completely independent. For a fixed $\gamma$, let $\mathcal{E}_{\gamma}$ denote the event that for at least $(3 / 4)\left|Y_{\gamma}\right|$ of values $(y, \gamma): y \in Y_{\gamma}$, the event $\eta_{\mathcal{S}^{\prime}}(y, \psi, \gamma)$ happens. Also, let $\mathcal{E}$ denote the event that for at least $(3 / 4) \Gamma$ choices of $\gamma$, the event $\mathcal{E}_{\gamma}$ occurs. Then

$$
\begin{gathered}
\operatorname{Pr}\left[\mathcal{E}_{\gamma}\right] \leq 2^{\left|Y_{\gamma}\right|} \cdot 2^{-(3 / 4)\left|Y_{\gamma}\right| \lambda / 2} \leq 2^{-(1 / 64)|Y| \lambda} \\
\operatorname{Pr}[\mathcal{E}] \leq 2^{\Gamma} \cdot\left(2^{-(1 / 64)|Y| \lambda}\right)^{3 \Gamma / 4} \leq 2^{-(\Gamma|Y| \lambda) / 128}
\end{gathered}
$$

If event $\mathcal{E}$ does not occur, then at least $(3 \Gamma / 4)(3|Y| / 64) \geq(\Gamma|Y|) / 32$ of the source-sink pairs are not disconnected, and thus $\operatorname{Pr}\left[\eta_{\mathcal{S}^{\prime}}(\psi)\right] \leq 2^{-\Gamma|Y| \lambda / 128}$.

To bound the probability of $\eta(\psi)$, we use the union-bound over all the possible subsets $\mathcal{S}^{\prime} \subseteq V_{\psi}$. Recall that $V_{\psi}$ is the union of at most $D$ sets $V(x), x \in \psi$, and that each set $V(x)$ contains $|Y| Z|F| C$ vertices. Therefore, the number of such subsets $\mathcal{S}$ is at most:

$$
2^{D|Y| Z|F| C}=2^{|Y| D \cdot 64|F|^{D} \cdot|F| \cdot 4 \lambda|F|^{D}}=2^{2^{8} D|F|^{2 D+1} \lambda|Y|}
$$

Since $\Gamma=2^{20} D|F|^{2 D+1}$, by using the union bound, the probability of $\eta(\psi)$ is bounded by $1 / 2 m$.

Finally, using the union bound over all $\psi \in \mathcal{G}$, we get the following corollary from the above lemma.

Corollary 1 With probability at least $1 / 2$, the event $\eta(\psi)$ does not happen for any good constraint $\psi \in \mathcal{G}$.

We have thus proved the following theorem. 
Theorem 4.3 If we start with a NO-Instance of label-cover, then with probability at least $1 / 2$, the multicut instance has the property that any solution of weight at most $g C_{Y I} / 8$ where $g=2^{\Omega\left(\log ^{1-\epsilon} N\right)}$, leaves a $1 / 2^{8}$-fraction of source-sink pairs connected.

We say that a solution to a multicut instance is an $(\alpha, \beta)$ bicriteria approximation for some $0 \leq$ $\alpha \leq 1$ and $\beta \geq 1$, if it disconnects at least an $\alpha$ fraction of the pairs and deletes at most $\beta$ OPT edges, where OPT denotes the cost of the optimal solution for the multicut instance. We can thus conclude that there does not exist a $\left(1-1 / 2^{8}, 2^{\Omega\left(\log ^{1-\epsilon} N\right)}\right)$ bicriteria approximation algorithm for directed multicut, for any constant $\epsilon>0$, unless NP $\subseteq$ co-RP. Using the poly-length and polytime verifiable witness property of NP, a standard argument can be used to convert this in to a ZPP algorithm. Thus directed multicut is hard to approximate to with in $2^{\Omega\left(\log ^{1-\epsilon} N\right)}$ even for the bicriteria version unless $\mathrm{NP} \subseteq \mathrm{ZPP}$.

\section{Table of Parameters}

Notations and parameters for the label cover instance

\begin{tabular}{|l|l|l|}
\hline$X$ & Set of variables & $|X|=n$ \\
\hline$\Psi$ & Set of constraints & $|\Psi|=m=\operatorname{poly}(n)$ \\
\hline$D=c_{1}$ & Number of variables per constraint & $c_{1}$ is some constant \\
\hline$F,|F|=O\left(2^{(\log m)^{1-\epsilon_{0}}}\right)$ & Alphabet over which variables take values & $\epsilon_{0}$ can be any constant \\
\hline$k_{\psi}=\left|R_{\psi}\right| \leq|F|^{D}$ & Number of satisfying assignments for $\psi$ & \\
\hline $\begin{array}{c}g=\Theta\left(\frac{|F|^{1 / D}}{D}\right) \\
=\Omega\left(2^{(\log m)^{1-\epsilon}}\right)\end{array}$ & The hardness gap & $\epsilon$ is a constant depending on $\epsilon_{0}$ \\
\hline
\end{tabular}

Parameters for the multicut construction

\begin{tabular}{|l|l|}
\hline$\lambda=O(\log n)$ & Number of copies of each variable-answer pair per set $S_{j}(y, \psi, \gamma)$ \\
\hline$C=4 \lambda|F|^{D}<$ poly $(n)$ & Number of vertices representing copies of each variable-answer pair in graph $H$ \\
\hline$\tau=\Gamma m$ & Parameter of the labeling scheme \\
\hline$Z=64|F|^{D}$ & Parameter of the labeling scheme (number of layers) \\
\hline$Y$ & Set of labels, $|Y|=O\left(\tau^{3} Z^{2}\right)$ \\
\hline$\Gamma=2^{20} D|F|^{2 D+1}$ & Repetition parameter. \\
\hline
\end{tabular}

\subsection{Hardness of Directed Sparsest Cut}

We can now use an argument similar to the one given in [11] for undirected cut problems, to extend the above bicriteria hardness for multicut to a matching hardness result for directed sparsest cut. For sake of completeness, we briefly sketch the argument. Let $\alpha_{0}=1-1 / 2^{8}$ and let $\beta_{0}=2^{\Omega\left(\log ^{1-\epsilon} N\right)}$ denote the hardness factor obtained above. Also, let OPT denote the optimal value for a given multicut instance. Suppose we have a $\beta^{\prime}$-approximation algorithm for sparsest cut. Let $K$ denote the total number of source-sink pairs in the multicut instance above. Then as long as at least $\left(1-\alpha_{0}\right) K$ pairs remain to be separated, we can use the $\beta^{\prime}$-approximation to find a subset $E^{\prime}$ of edges of size at most $\beta^{\prime}\left(\frac{\mathrm{OPT}}{\left(1-\alpha_{0}\right) K}\right) p$ that separates at least $p$ pairs for some integer $p \geq 1$. We delete the edges in $E^{\prime}$ and repeat this process until the number of remaining pairs falls below $\left(1-\alpha_{0}\right) K$. It is easy to see that the total number of edges deleted over all iterations is at most $\left(\frac{\beta^{\prime}}{\left(1-\alpha_{0}\right)}\right)$ OPT. The bicriteria hardness for directed multicut implies that we must have $\beta^{\prime} \geq\left(1-\alpha_{0}\right) \beta_{0}=2^{\Omega\left(\log ^{1-\epsilon} N\right)}$. This concludes the proof of Theorem 1.2. 


\subsection{Stronger PCP Characterization and Polynomial Hardness}

We notice that the existence of PCP's with proof table entries ranging over some alphabet $F$ whose size is polynomial, logarithmic number of random bits, constant number of queries, perfect completeness and polynomially small soundness would imply a stronger version of Theorem 4.1, which would in turn imply, using the same arguments as in the proof of Theorem 4.2, that labelcover is hard to approximate up to some polynomial factor $g$. We can then use our reduction to show polynomial hardness of approximation for directed multicut and directed sparsest cut.

\section{APX-Hardness of Undirected Sparsest Cut}

We prove here that undirected non-uniform sparsest cut problem is APX-hard even on planar graphs (Theorem 1.4). We will show this result by giving a reduction from the max cut problem on cubic graphs. In this version of the max cut problem, we are given a graph $G=(V, E)$ where the degree of each vertex is between 1 and 3 . The goal is to find a bipartition $(S, \bar{S})$ of the vertices such that the number of edges crossing the cut, denoted $|E(S, \bar{S})|$, is maximized. Alimonti and Kann [2] proved that max cut on cubic graphs is APX-hard. Specifically, they showed the following result:

Theorem 5.1 [2] There exist constants $\epsilon, \gamma$ with $0<\epsilon<1 / 2<\gamma$, such that it is NP-hard to decide whether a given cubic graph $G(V, E)$ has max cut value at least $\gamma|E|$ (a Yes-INSTANCE) or at most $(1-\epsilon) \gamma|E|$ (a No-Instance).

Let $G=(V, E)$ be an instance of max cut where $G$ is a cubic graph. Let $|V|=n$ and $|E|=m$; clearly $n / 2 \leq m \leq 3 n / 2$ since $G$ is cubic. We define an instance $G^{\prime}=\left(V^{\prime}, E^{\prime}\right)$ of sparsest cut, as follows. The vertex set is the same as in $G$, except that we add two special vertices $s$ and $t$, i.e., $V^{\prime}=V \cup\{s, t\}$. The set of edges is: $E^{\prime}=\{(s, v) \mid v \in V\} \cup\{(t, v) \mid v \in V\}$. Finally, we define the source-sink pairs. For all $u, v \in V$ such that $(u, v) \in E$, we include $u, v$ as a source-sink pair. In addition, we include $6 \mathrm{~m}$ identical source-sink pairs with vertex $s$ as the source and the vertex $t$ as the sink. It is easy to see that the graph $G^{\prime}$ is planar: a natural planar embedding of $G^{\prime}$ is to arrange all vertices in $V$ along a line $L$ and place $s$ and $t$ on opposite sides of line $L$.

The following claim completes the proof of Theorem 1.4.

Claim 5.1 If $G$ is a YES-INSTANCE of max cut problem, then there is a cut in $G^{\prime}$ of sparsity at most $\alpha=\frac{n}{6 m+\gamma m}$. If $G$ is a No-INSTANCE of max cut problem, then the sparsity of any cut in $G^{\prime}$ is at least $(1+\epsilon / 18) \alpha$.

Proof: Suppose $G$ is a Yes-Instance. Let $(S, \bar{S})$ be the optimal solution to the max cut problem on $G$, where $|E(S, \bar{S})| \geq \gamma m$. We define a cut $\left(S^{\prime}, \overline{S^{\prime}}\right)$ in graph $G^{\prime}$ with $S^{\prime}=\{s\} \cup S$. Notice that $\left|E\left(S^{\prime}, \overline{S^{\prime}}\right)\right|=n$, since for every $v \in V$, exactly one of the two edges $(s, v),(t, v)$ crosses the cut. Total number of pairs separated is at least $6 m+\gamma m$. Hence the sparsity of cut $\left(S^{\prime}, \overline{S^{\prime}}\right)$ is at most $\alpha=\frac{n}{6 m+\gamma m}$.

Assume now that $G$ is a No-Instance. We show that the sparsity of any cut in $G^{\prime}$ is at least $(1+\epsilon / 18) \alpha$. Consider any cut $\left(S^{\prime}, \overline{S^{\prime}}\right)$ in $G^{\prime}$. We distinguish between two cases.

The first case is when $s$ and $t$ both lie on the same side of the cut, say $S^{\prime}$. Let $\left|\overline{S^{\prime}}\right|=k$. Then the number of edges crossing the cut is exactly $2 k$, while the number of pairs separated by the cut is at most $3 k$ (since $G$ is a cubic graph). Thus the cut sparsity is at least: $\frac{2 k}{3 k}=\frac{2}{3}$. On the other hand, $\alpha=\frac{n}{6 m+\gamma m} \leq \frac{1}{3(1+\gamma / 2)}<\frac{4}{15}$, since $m \geq n / 2$ and $\gamma>1 / 2$. It follows that the cut sparsity is at least $(1+\epsilon / 18) \alpha$. 
It now only remains to analyze the second case, where $s$ and $t$ belong to different sides of the cut. Assume w.l.o.g. that $s \in S^{\prime}$. No matter how the rest of the vertices are partitioned, the number of edges crossing the cut is exactly $n$. We define a cut $(S, \bar{S})$ in $G$ as follows: $S=S^{\prime} \backslash\{s\}$. Since $G$ is a No-Instance, the number of edges crossing $(S, \bar{S})$ in $G$ is at most $(1-\epsilon) \gamma m$. Thus the total number of pairs separated by $\left(S^{\prime}, \overline{S^{\prime}}\right)$ is at most $6 m+(1-\epsilon) \gamma m$, and the sparsity of the cut is at least: $\alpha^{\prime}=\frac{n}{6 m+(1-\epsilon) \gamma m}$. It now only remains to show that $\frac{\alpha^{\prime}}{\alpha} \geq \frac{6 m+\gamma m}{6 m+(1-\epsilon) \gamma m} \geq 1+\epsilon / 18$, which is easily verified using the fact that $\gamma>\frac{1}{2}$.

\section{Acknowledgements}

We would like to thank Chandra Chekuri, Irit Dinur, Piotr Indyk, and Ran Raz for helpful discussions. We would also like to thank the anonymous reviewers whose comments have helped improve the presentation. 


\section{References}

[1] A. Agarwal, N. Alon, and M. Charikar. Improved approximation for directed cut problems. In Proc. of STOC, 2007, pp. $671-680$.

[2] P. Alimonti and V. Kann. Hardness of approximating problems on cubic graphs. Theoretical Computer Science, 237: 123-134, 2000.

[3] C. Ambühl, M. Mastrolilli, and O. Svensson, Inapproximability Results for Sparsest Cut, Optimal Linear Arrangement, and Precedence Constrained Scheduling. In Proc. of FOCS, 2007, pp. 329-337.

[4] M. Andrews and L. Zhang. Logarithmic hardness of the directed congestion minimization problem. In Proc. of STOC'06.

[5] S. Arora, J. R. Lee, A. Naor. Euclidean distortion and the sparsest cut. In Proc. of STOC, 2005 , pp. 553-562.

[6] S. Arora, C. Lund, R. Motwani, M. Sudan, and M. Szegedy. Proof verification and the hardness of approximation problems. Journal of the ACM, 45(3):501-555, 1998.

[7] S. Arora, S. Rao, U. V. Vazirani. Expander flows, geometric embeddings and graph partitioning. In Proc. of STOC, 2004, pp. 222-231.

[8] S. Arora and S. Safra. Probabilistic checking of proofs: A new characterization of NP. JACM, 45(1):70-122, 1998.

[9] M. Bellare, S. Goldwasser, C. Lund, A. Russeli: Efficient probabilistically checkable proofs and applications to approximations. In Proc. of STOC, 1993, pp. 294-304.

[10] M. Charikar, K. Makarychev, Y. Makarychev. Directed Metrics and Directed Graph Partitioning Problems. In Proc. of SODA, 2006, pp. 51-60.

[11] S. Chawla, R. Krauthgamer, R. Kumar, Y. Rabani, D. Sivakumar. On the Hardness of Approximating Multicut and Sparsest-Cut. in Proc. IEEE Conference on Computational Complexity, 2005, pp. 144-153.

[12] J. Cheriyan, H. J. Karloff, Y. Rabani. Approximating Directed Multicuts. In Proc. of FOCS, 2001, pp. 320-328.

[13] J. Chuzhoy and S. Khanna. Hardness of Cut Problems in Directed Graphs. In Proc. of STOC, 2006, pp. $527-536$.

[14] J. Chuzhoy and S. Khanna. Polynomial Flow-cut Gaps and Hardness of Directed Cut Problems. In Proc. of STOC, 2007, pp. 179-188.

[15] J. Chuzhoy and S. Khanna. Hardness of Directed Routing with Congestion. ECCC Technical Report TR06-109, August 2006. http://eccc.hpi-web.de/eccc-reports/2006/TR06109/index.html.

[16] J. Chuzhoy, V. Guruswami, S. Khanna, and K. Talwar. Hardness of Routing with Congestion in Directed Graphs. In Proc. of STOC, 2007. 
[17] E. Dahlhaus, D. S. Johnson, C. H. Papadimitriou, P. D. Seymour, M. Yannakakis. The Complexity of Multiterminal Cuts. In SIAM J. Comput., 23(4): 864-894, 1994.

[18] I. Dinur, E. Fischer, G. Kndler, R. Raz and S. Safra. PCP characterizations of NP: towards a polynomially-small error-probability. In Proceedings of the Thirty-First Annual ACM Symposium on Theory of Computing (STOC), pp. 29-40, 1999.

[19] I. Dinur and S. Safra. On the hardness of approximating label-cover. Information Processing Letters, 89(5), pp. 247-254, 2004.

[20] U. Feige. Relations between average case complexity and approximation complexity. In Proc. of STOC, 2002, pp. 534-543.

[21] U. Feige and S. Kogan. Hardness of Approximation of the Balanced Complete Bipartite Subgraph Problem. Technical Report MCS04-04, Department of Computer Science and Applied Math., The Weizmann Institute of Science, 2004.

[22] L. R. Ford, D. R. Fulkerson, 1962. Flows in Networks. Princeton University Press, Princeton, NJ.

[23] V. Guruswami and K. Talwar. Hardness of Low Congestion Routing in Directed Graphs. ECCC Technical Report TR06-141, November 2006, http://eccc.hpi-web.de/eccc-reports/2006/TR06141/index.html.

[24] N. Garg, V. Vazirani, M. Yannakakis. Approximate max-flow min-(multi)cut theorems and their applications. In Proc. of STOC, 1993, pp. 698-707.

[25] N. Garg, V. Vazirani, M. Yannakakis. Primal-Dual Approximation Algorithms for Integral Flow and Multicut in Trees. Algorithmica, 18(1):3-20, 1997. Preliminary version in Proc. of ICALP, 1993.

[26] A. Gupta. Improved results for directed multicut. In Proc. of SODA, 2003, pp. 454-455.

[27] M.T. Hajiaghayi, H. Räcke. An $O(\sqrt{n})$-Approximation Algorithm For Directed Sparsest Cut, Information Processing Letters, 97(4): 156-160, 2006.

[28] S. Khot. On the power of unique 2-prover 1-round games. In Proc. of STOC, 2002, pp. 767-775.

[29] S. Khot, N. K. Vishnoi. The Unique Games Conjecture, Integrality Gap for Cut Problems and the Embeddability of Negative Type Metrics into $\ell_{1}$. In Proc. of FOCS, 2005, pp. 53-62.

[30] Y. Kortsarts, G. Kortsarz and Z. Nutov. Greedy approximation algorithms for directed multicuts. Networks 45(4), pp. 214-217, 2005.

[31] T. Leighton and S. Rao. Multicommodity max-flow min-cut theorems and their use in designing approximation algorithms. JACM, 46(6):787-832, 1999. Preliminary version in Proc. of FOCS, 1988.

[32] R. Raz. A parallel repetition theorem. SIAM J. of Computing, 27(3):763-803, 1998.

[33] M. E. Saks, A. Samorodnitsky, L. Zosin. A Lower Bound On The Integrality Gap For Minimum Multicut In Directed Networks. Combinatorica 24(3): 525-530 (2004). 